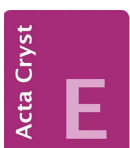

CRYSTALLOGRAPHIC COMMUNICATIONS

ISSN 2056-9890

Received 5 October 2017

Accepted 13 October 2017

Edited by A. V. Yatsenko, Moscow State University, Russia

Keywords: crystal structure; azacyclic allenes; thermolysis; microwave synthesis; (epiminomethano)cyclopenta[a]indene; 3-benzazepine; synchrotron X-ray diffraction.

CCDC reference: 1579823

Supporting information: this article has supporting information at journals.iucr.org/e

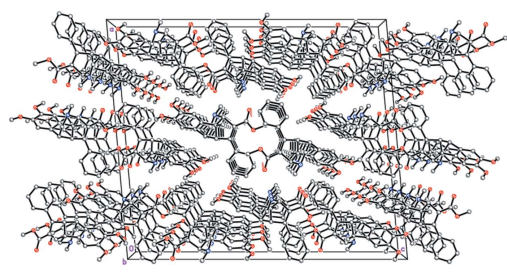

OPEN $\odot$ ACCESS

\section{Unusual thermolysis of azacyclic allene under microwave conditions: crystal structure of (3RS,3aSR,8RS,8aRS)-methyl 5,6-dimethoxy-3a,10- dimethyl-1-phenyl-3,3a,8,8a-tetrahydro-3,8- (epiminomethano)cyclopenta[a]indene-2-carboxyl- ate from synchrotron X-ray diffraction}

\author{
Le Tuan Anh, ${ }^{a *}$ Alexander A. Titov, ${ }^{b}$ Maxim S. Kobzev, ${ }^{b}$ Leonid G. Voskressensky, ${ }^{b}$ \\ Alexey V. Varlamov, ${ }^{b}$ Pavel V. Dorovatovskii ${ }^{c}$ and Victor N. Khrustalev ${ }^{d}$

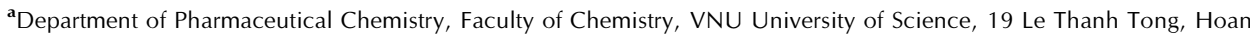 \\ Kiem, Hanoi 100000, Vietnam, 'Organic Chemistry Department, Peoples' Friendship University of Russia, (RUDN \\ University), Miklukho-Maklay St., 6, Moscow 117198, Russian Federation, 'National Research Centre "Kurchatov \\ Institute", 1 Acad. Kurchatov Sq., Moscow 123182, Russian Federation, and 'Inorganic Chemistry Department, Peoples' \\ Friendship University of Russia, (RUDN University), Miklukho-Maklay St., 6, Moscow 117198, Russian Federation. \\ *Correspondence e-mail: huschemical.lab@gmail.com
}

The title compound, $\mathrm{C}_{25} \mathrm{H}_{27} \mathrm{NO}_{4}(\mathrm{I})$, the product of the unusual thermolysis of azacyclic allene methyl 10,11-dimethoxy-3,8-dimethyl-6-phenyl-3-azabenzo$[d]$ cyclodeca-4,6,7-triene-5-carboxylate, represents a bicyclic heterosystem and crystallizes in the monoclinic space group $P 2_{1} / c$ with three crystallographically independent molecules in the unit cell. These independent molecules adopt very similar geometries and differ only in the conformations of the two methoxy substituents on the benzene ring. In two of the three independent molecules, both methoxy groups are almost coplanar with the benzene ring [the $\mathrm{C}-\mathrm{C}-$ $\mathrm{O}-\mathrm{Me}$ torsion angles are 10.8 (2), 12.3 (2), 9.1 (2) and $\left.13.6(3)^{\circ}\right]$, whereas in the third molecule, one of the methoxy groups is practically coplanar to and the other methoxy group is roughly perpendicular to the benzene ring, the $\mathrm{C}-\mathrm{C}-$ $\mathrm{O}-\mathrm{Me}$ torsion angles being $14.1(2)$ and $76.5(2)^{\circ}$. The molecule of (I) comprises a fused tetracyclic system containing two five-membered rings (cyclopentenes) and two six-membered rings (piperidine and benzene). The five-membered rings have the usual envelope conformation, with the methylsubsituted $\mathrm{C}$ atom as the flap in each molecule, and the six-membered piperidine ring has a chair conformation. The methyl substituent at the $\mathrm{N}$ atom occupies the sterically favourable equatorial position. The carboxylate group lies almost within the basal plane of the parent cyclopentene ring [making dihedral angle of $11.68(8), 18.94(9)$ and $15.16(9)^{\circ}$ in the three independent molecules], while the phenyl substituent is twisted by 48.26 (6), 42.04 (6) and 41.28 (6) (for the three independent molecules) relative to this plane. In the crystal, molecules of (I) form stacks along the $b$-axis direction. The molecules are arranged at van der Waals distances.

\section{Chemical context}

The allene fragment is a part of some natural compounds steroids, prostaglandins, amines acids, nucleosides. The vast majority of them contain a non-cyclic allene fragment. Tenmembered steroids with an allene fragment in bacteria have already been studied (Batzold \& Robinson, 1975, 1976; Batzold et al., 1977). Bicyclic lactones comprising tenmembered sesquiterpene allenes have been isolated from the Vernonia plants of South America (Bohlmann et al., 1980; 
Jakupovic et al., 1985; Warning et al., 1987). Natural functionalized allenes are inhibitors of enzymes that exhibit cytotoxic and antiviral activity (Krause \& Hoffmann-Röder, 2004). However, heterocyclic allene systems are practically unexplored. Nitrogen-containing nonatrienes-3,6,7 were obtained for the first time in 1984 with a yield of $8-10 \%$ (Sashida \& Tsuchiya, 1984). Further, the synthesis of 1-azadeca(undeca)4,5-dien-2-ones has been carried out (Perscheid et al., 2011).

Recently, we proposed a relatively simple synthesis of benzoazadecatrienes-4,6,7 from $1 R$-1-phenylethynyl tetrahydroisoquinolines and activated terminal alkynes in trifluoroethanol at $256 \mathrm{~K}$ (Voskressensky et al., 2017). However, the thermal transformations of such strained systems have not yet been studied.

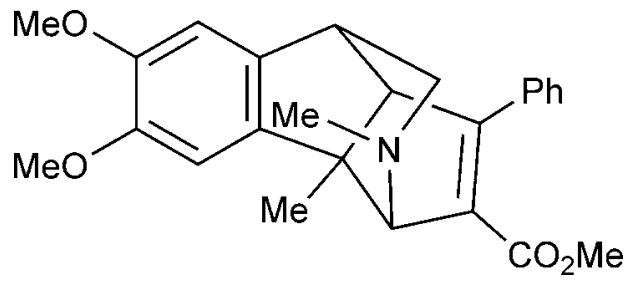

In this work, the thermolysis of an azacyclic allene under microwave conditions was carried out by our research group. The structure of the product of this unusual reaction was established unambiguously by synchrotron X-ray diffraction study.

\section{Structural commentary}

The title compound crystallizes with three crystallographically independent molecules ( $A, B$ and $C$, containing atoms $\mathrm{N} 10$, $\mathrm{N} 32$ and N54, respectively) in the unit cell (Fig. 1). These independent molecules adopt very similar geometries and differ only in the conformations of the two methoxy substituents at the benzene ring. In two of the three independent molecules, both methoxy groups are almost coplanar to the benzene ring [the $\mathrm{C}-\mathrm{C}-\mathrm{O}-\mathrm{Me}$ torsion angles are 10.8 (2) and $12.3(2)^{\circ}$ in molecule $A$ and 9.1 (2) and $13.6(3)^{\circ}$ in $B$ ], whereas in the third molecule, $C$, one of the methoxy groups is practically coplanar with and the other methoxy group is roughly perpendicular to the benzene ring, the $\mathrm{C}-\mathrm{C}-\mathrm{O}-\mathrm{Me}$ torsion angles being 14.1 (2) and $76.5(2)^{\circ}$ ].

The molecule of (I) comprises a fused tetracyclic system containing two five-membered rings (cyclopentenes) and two six-membered rings (piperidine and benzene) (Fig. 1). The five-membered rings have the usual envelope conformation, with the methyl-subsituted $\mathrm{C}$ atom as the flap in each molecule, and the six-membered piperidine ring has a chair conformation. The methyl substituent at the nitrogen atom occupies the sterically favourable equatorial position. The carboxylate group lies almost within the basal plane of the parent cyclopentene ring, making dihedral angles of 11.68 (8), 18.94 (9) and $15.16(9)^{\circ}$, respectively, in molecules $A, B$ and $C$, while the phenyl substituent is twisted by 48.26 (6), 42.04 (6) and $41.28(6)^{\circ}$ relative to this plane in molecules $A, B$ and $C$, respectively.

The title molecule possesses four asymmetric centers at the C3, C3A, C8 and C8A carbon atoms and can have potentially numerous diastereomers. The crystal of (I) is racemic and consists of enantiomeric pairs with the following relative configuration of the centers: $r a c-3 R^{*}, 3 \mathrm{a} S^{*}, 8 R^{*}, 8 \mathrm{a} R^{*}$.

\section{Supramolecular features}

The crystal packing motif of molecules of (I) is stacking along the crystallographic $b$ axis (Fig. 2). The molecules are arranged at van der Waals distances.

\section{Synthesis and crystallization}

A stirred solution of cyclic allene $(0.13 \mathrm{~g}, 0.32 \mathrm{mmol})$ in toluene $(5 \mathrm{~mL})$ was heated at $453 \mathrm{~K}$ for $1 \mathrm{~h}$ in a microwave reactor (Anton Paar Monowave 300) (Fig. 3). The solvent was evaporated in vacuo, and the residue recrystallized from ether to give $60 \mathrm{mg}$ of colourless crystals of (I) in a yield of $50 \%$, m.p. $=422-424 \mathrm{~K}$ (ether).

${ }^{1} \mathrm{H}$ NMR $\left(\mathrm{CDCl}_{3}, 600 \mathrm{MHz}\right): \delta=1.47$ (3H, s, 3a-Me), 2.21 $(3 \mathrm{H}, s, \mathrm{NMe}), 2.47(1 \mathrm{H}, d d, J=2.5 \mathrm{~Hz}, J=10.7 \mathrm{~Hz}, 9-\mathrm{CH}), 2.65$ $(1 \mathrm{H}, d d, J=2.5 \mathrm{~Hz}, J=10.7 \mathrm{~Hz}, 9-\mathrm{CH}), 3.09-3.10(1 \mathrm{H}, m$, 8-CH), $3.22(1 \mathrm{H}, d, J=5.0 \mathrm{~Hz}, 8 \mathrm{a}-\mathrm{CH}), 3.67(1 \mathrm{H}, d, J=1.7 \mathrm{~Hz}$, 3-CH), 3.69 (3H, s, OMe), 3.88 (3H, s, OMe), 3.91 (3H, $s$, OMe), $6.73(1 \mathrm{H}, s, \mathrm{H} 7), 6.74(1 \mathrm{H}, s, \mathrm{H} 4), 7.35-7.40(3 \mathrm{H}, m$, $\mathrm{Ph}), 7.47-7.48$ (2H, $m, \mathrm{Ph}) ;{ }^{13} \mathrm{C}$ NMR (DMSO- $\left.d_{6}, 150 \mathrm{MHz}\right): \delta$ $=18.3,42.3$, 42.6, 51.1, 51.2, 55.7, 55.8, 59.6, 67.9, 72.8, 106.9, 107.1, 124.9, 127.9 (2 C), 128.0 (2 C), 128.5, 135.1, 137.1, 137.3, $147.8,148.3,151.1,166.3 ; m / z: 406[M+\mathrm{H}]^{+}$. Analysis calculated

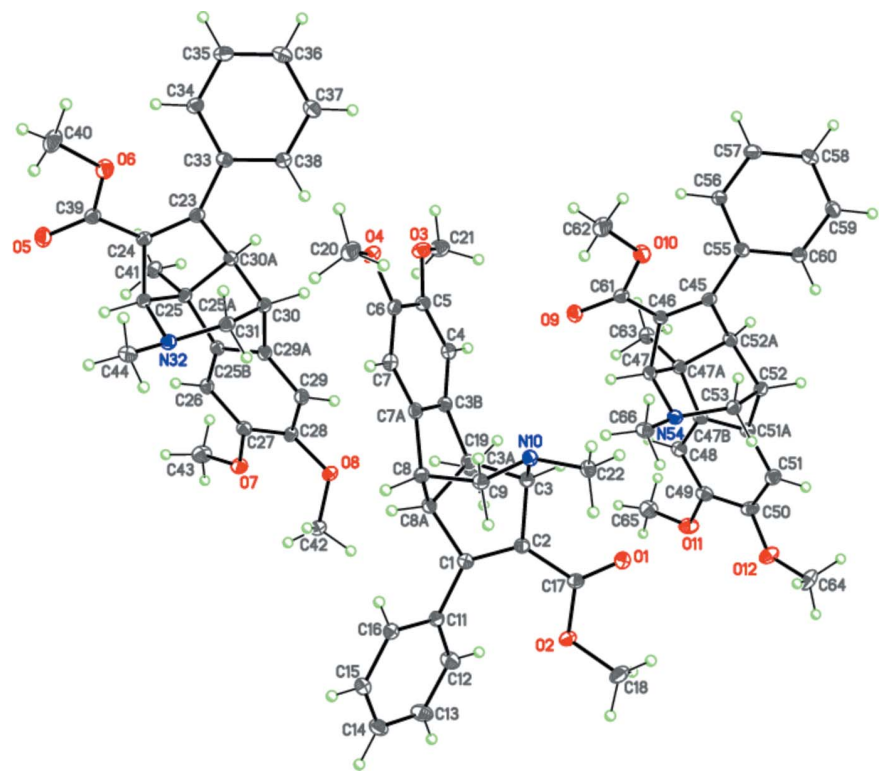

Figure 1

The molecular structure of (I) showing the three crystallographically independent molecules. Displacement ellipsoids are shown at the $50 \%$ probability level. $\mathrm{H}$ atoms are shown as small spheres of arbitrary radius. 


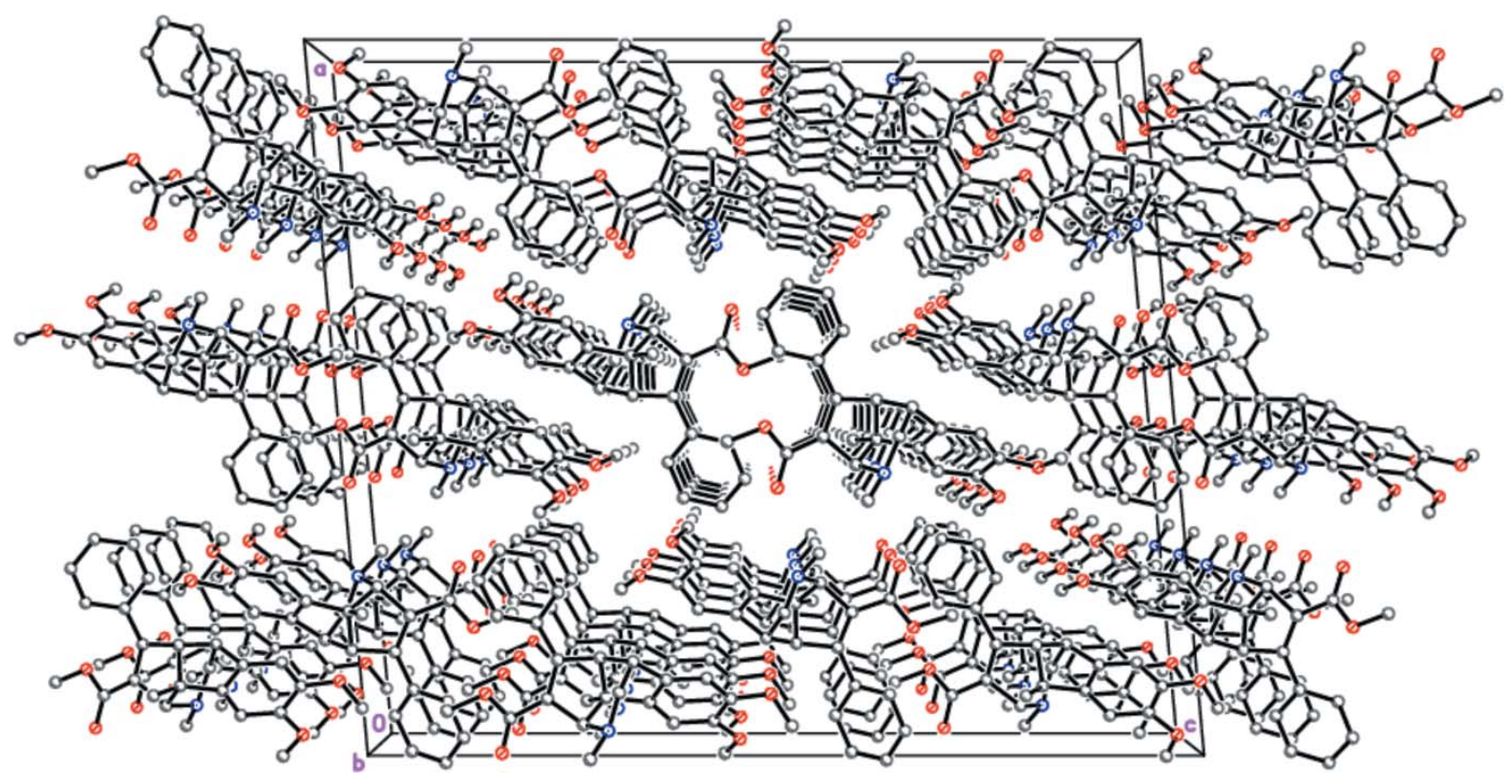

Figure 2

Crystal structure of (I) illustrating the stacks along [010]. For clarity, $\mathrm{H}$ atoms have been omitted.

for $\mathrm{C}_{25} \mathrm{H}_{27} \mathrm{NO}_{4}$ (\%): C 74.03, $\mathrm{H}$ 6.71, N 3.45; found (\%): C 74.04, H 6.71, N 3.41 .

\section{Refinement}

Crystal data, data collection and structure refinement details are summarized in Table 1 . The X-ray diffraction study was carried out on the 'Belok' beamline of the National Research Center 'Kurchatov Institute' (Moscow, Russian Federation) using a Rayonix SX165 CCD detector. A total of 720 images were collected using an oscillation range of $1.0^{\circ}$ ( $\varphi$ scan mode, two different crystal orientations) and corrected for absorption using the Scala program (Evans, 2006). The data were indexed, integrated and scaled using the utility $i$ MOSFLM in CCP4 (Battye et al., 2011).

Hydrogen atoms were placed in calculated positions with $\mathrm{C}-\mathrm{H}=0.95-1.00 \AA$ and refined in the riding model with fixed isotropic displacement parameters $\left[U_{\text {iso }}(\mathrm{H})=1.5 U_{\text {eq }}(\mathrm{C})\right.$ for the $\mathrm{CH}_{3}$-groups and $1.2 U_{\text {eq }}(\mathrm{C})$ for the other groups].

A rather large number of reflections have been omitted for the following reasons: (i) In order to achieve better I $\sigma$ statistics for high-angle reflections, we selected exposure times so as to admit a minor fraction of intensity overloads in the low-angle part of the detector. These low-angle reflections with imprecisely measured intensities were excluded from the
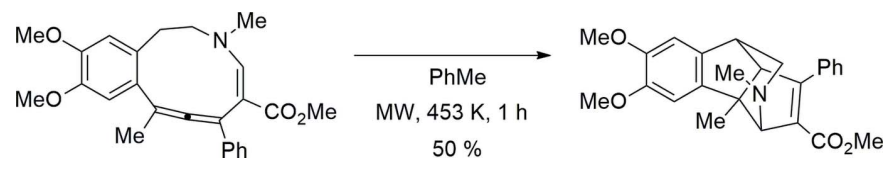

Figure 3

Thermolysis of azacyclic allene methyl 10,11-dimethoxy-3,8-dimethyl-6phenyl-3-azabenzo[d]cyclodeca-4,6,7-triene-5-carboxylate under microwave conditions. final steps of the refinement. (ii) In the present setup of the synchrotron diffractometer, the low-temperature device eclipses a small region of the $2 \mathrm{D}$ detector near the high-angle limit. This small shadowed region was not masked during integration of the diffraction frames, which erroneously resulted in zero intensity for some reflections.

Table 1

Experimental details.

\begin{tabular}{ll}
\hline Crystal data & \\
Chemical formula & $\mathrm{C}_{25} \mathrm{H}_{27} \mathrm{NO}_{4}$ \\
$M_{\mathrm{r}}$ & 405.47 \\
Crystal system, space group & Monoclinic, $P{ }_{1} / c$ \\
Temperature $(\mathrm{K})$ & 100 \\
$a, b, c(\AA)$ & $26.404(5), 7.9303(16), 30.720(6)$ \\
$\beta\left({ }^{\circ}\right.$ & $95.09(3)$ \\
$V\left(\AA^{3}\right)$ & $6407(2)$ \\
$Z$ & 12 \\
Radiation type & Synchrotron, $\lambda=0.96990 \AA$ \\
$\mu\left(\mathrm{mm}^{-1}\right)$ & 0.18 \\
Crystal size $(\mathrm{mm})$ & $0.25 \times 0.24 \times 0.21$
\end{tabular}

Data collection

Diffractometer

Absorption correction

$T_{\min }, T_{\max }$

No. of measured, independent and observed $[I>2 \sigma(I)]$ reflections

$R_{\text {int }}$

$(\sin \theta / \lambda)_{\max }\left(\AA^{-1}\right)$

Rayonix SX165 CCD

Multi-scan (SCALA; Evans, 2006) $0.940,0.951$

74044, 13622, 10086

0.071

0.659

Refinement

$R\left[F^{2}>2 \sigma\left(F^{2}\right)\right], w R\left(F^{2}\right), S$

No. of reflections

No. of parameters

$\mathrm{H}$-atom treatment

$\Delta \rho_{\max }, \Delta \rho_{\min }\left(\mathrm{e} \AA^{-3}\right)$

$0.053,0.146,1.07$

13622

827

$\mathrm{H}$-atom parameters constrained $0.46,-0.38$

Computer programs: Marccd (Doyle, 2011), iMOSFLM (Battye et al., 2011), SHELXT (Sheldrick, 2015a), SHELXL2014 (Sheldrick, 2015b) and SHELXTL (Sheldrick, 2008). 


\section{Funding information}

The publication was prepared with the support of the RUDN University Program ' $5-100$ ' and by the Russian Foundation for Basic Research (project No. 17-53-540001-Viet-a). This research is funded by the Vietnam National Foundation for Science and Technology Development (NAFOSTED) under grant No. 104.01-2015.27. Synchrotron radiation measurements were performed at the unique scientific facility Kurchatov Synchrotron Radiation Source supported by the Ministry of Education and Science of the Russian Federation (project code RFMEFI61917X0007).

\section{References}

Battye, T. G. G., Kontogiannis, L., Johnson, O., Powell, H. R. \& Leslie, A. G. W. (2011). Acta Cryst. D67, 271-281.

Batzold, F. H., Covey, D. F. \& Robinson, C. H. (1977). Cancer Treat. Rep. 61, 255-257.

Batzold, F. H. \& Robinson, C. H. (1975). J. Am. Chem. Soc. 97, 25762578.
Batzold, F. H. \& Robinson, C. H. (1976). J. Org. Chem. 41, 313-317. Bohlmann, F., Gupta, R. K., Jakupovic, J., King, R. M. \& Robinson, H. (1980). Liebigs Ann. Chem. pp. 1904-1906.

Doyle, R. A. (2011). Marccd software manual. Rayonix L. L. C., Evanston, IL 60201, USA.

Evans, P. (2006). Acta Cryst. D62, 72-82.

Jakupovic, J., Schmeda-Hirschmann, G., Schuster, A., Zdero, C., Bohlmann, F., King, R. M., Robinson, H. \& Pickardt, J. (1985). Phytochemistry, 25, 145-158.

Krause, N. \& Hoffmann-Röder, A. (2004). Modern Allene Chemistry, Vol. 2, edited by N. Krause \& A. S. K. Hashmi, pp. 9971032. Weinheim: Wiley-VCH Verlag GmbH \& Co.

Perscheid, M., Schollmeyer, D. \& Nubbemeyer, U. (2011). Eur. J. Org. Chem. pp. 5250-5253.

Sashida, H. \& Tsuchiya, T. (1984). Chem. Pharm. Bull. 32, 4600-4607. Sheldrick, G. M. (2008). Acta Cryst. A64, 112-122.

Sheldrick, G. M. (2015a). Acta Cryst. A71, 3-8.

Sheldrick, G. M. (2015b). Acta Cryst. C71, 3-8.

Voskressensky, L. G., Titov, A. A., Dzhankaziev, M. S., Borisova, T. N., Kobzev, M. S., Dorovatovskii, P. V., Khrustalev, V. N., Aksenov, A. V. \& Varlamov, A. V. (2017). New J. Chem. 41, 19021904.

Warning, U., Jakupovic, J., Bohlmann, F. \& Jones, S. B. (1987). Liebigs Ann. Chem. pp. 467-468. 


\section{supporting information}

Acta Cryst. (2017). E73, 1770-1773 [https://doi.org/10.1107/S2056989017014864]

Unusual thermolysis of azacyclic allene under microwave conditions: crystal structure of (3RS,3aSR,8RS,8aRS)-methyl 5,6-dimethoxy-3a,10-dimethyl-1phenyl-3,3a,8,8a-tetrahydro-3,8-(epiminomethano)cyclopenta[a]indene-2carboxylate from synchrotron $\mathrm{X}$-ray diffraction

Le Tuan Anh, Alexander A. Titov, Maxim S. Kobzev, Leonid G. Voskressensky, Alexey V. Varlamov, Pavel V. Dorovatovskii and Victor N. Khrustalev

Computing details

Data collection: Marccd (Doyle, 2011); cell refinement: iMOSFLM (Battye et al., 2011); data reduction: iMOSFLM (Battye et al., 2011); program(s) used to solve structure: SHELXT (Sheldrick, 2015a); program(s) used to refine structure: SHELXL2014 (Sheldrick, 2015b); molecular graphics: SHELXTL (Sheldrick, 2008); software used to prepare material for publication: SHELXTL (Sheldrick, 2008).

(3RS,3aSR,8RS,8aRS)-Methyl 5,6-dimethoxy-3a,10-dimethyl-1-phenyl-3,3a,8,8a-

tetrahydro-3,8-(epiminomethano)cyclopenta[a]indene-2-carboxylate

Crystal data

$\mathrm{C}_{25} \mathrm{H}_{27} \mathrm{NO}_{4}$

$M_{r}=405.47$

Monoclinic, $P 2_{1} / c$

$a=26.404(5) \AA$

$b=7.9303$ (16) $\AA$

$c=30.720(6) \AA$

$\beta=95.09(3)^{\circ}$

$V=6407(2) \AA^{3}$

$Z=12$

Data collection

Rayonix SX165 CCD diffractometer

/f scan

Absorption correction: multi-scan

(Scala; Evans, 2006)

$T_{\min }=0.940, T_{\max }=0.951$

74044 measured reflections
$F(000)=2592$

$D_{\mathrm{x}}=1.261 \mathrm{Mg} \mathrm{m}^{-3}$

Synchrotron radiation, $\lambda=0.96990 \AA$

Cell parameters from 600 reflections

$\theta=3.6-36.0^{\circ}$

$\mu=0.18 \mathrm{~mm}^{-1}$

$T=100 \mathrm{~K}$

Prism, colourless

$0.25 \times 0.24 \times 0.21 \mathrm{~mm}$

13622 independent reflections 10086 reflections with $I>2 \sigma(I)$

$R_{\text {int }}=0.071$

$\theta_{\text {max }}=39.7^{\circ}, \theta_{\min }=3.5^{\circ}$

$h=-33 \rightarrow 33$

$k=-8 \rightarrow 10$

$l=-39 \rightarrow 39$ 


\section{Refinement}

Refinement on $F^{2}$

Least-squares matrix: full

$R\left[F^{2}>2 \sigma\left(F^{2}\right)\right]=0.053$

$w R\left(F^{2}\right)=0.146$

$S=1.07$

13622 reflections

827 parameters

0 restraints

Primary atom site location: difference Fourier map

Secondary atom site location: difference Fourier map
Hydrogen site location: inferred from neighbouring sites

$\mathrm{H}$-atom parameters constrained

$w=1 /\left[\sigma^{2}\left(F_{\mathrm{o}}^{2}\right)+(0.0696 P)^{2}+0.8571 P\right]$

where $P=\left(F_{\mathrm{o}}^{2}+2 F_{\mathrm{c}}{ }^{2}\right) / 3$

$(\Delta / \sigma)_{\max }=0.001$

$\Delta \rho_{\max }=0.46 \mathrm{e} \AA^{-3}$

$\Delta \rho_{\text {min }}=-0.38$ e $\AA^{-3}$

Extinction correction: SHELXL2014 (Sheldrick, 2015b), $\mathrm{Fc}^{*}=\mathrm{kFc}\left[1+0.001 \times \mathrm{xc}^{2} \lambda^{3} / \sin (2 \theta)\right]^{-1 / 4}$

Extinction coefficient: 0.00120 (18)

\section{Special details}

Geometry. All esds (except the esd in the dihedral angle between two 1.s. planes) are estimated using the full covariance matrix. The cell esds are taken into account individually in the estimation of esds in distances, angles and torsion angles; correlations between esds in cell parameters are only used when they are defined by crystal symmetry. An approximate (isotropic) treatment of cell esds is used for estimating esds involving l.s. planes.

Fractional atomic coordinates and isotropic or equivalent isotropic displacement parameters $\left(\AA^{2}\right)$

\begin{tabular}{|c|c|c|c|c|}
\hline & $x$ & $y$ & $z$ & $U_{\text {iso }} * / U_{\text {eq }}$ \\
\hline $\mathrm{O} 1$ & $0.27574(4)$ & $0.67806(16)$ & $0.64919(4)$ & $0.0291(3)$ \\
\hline $\mathrm{O} 2$ & $0.19523(4)$ & $0.75446(16)$ & $0.65782(4)$ & $0.0293(3)$ \\
\hline $\mathrm{O} 3$ & $0.30254(4)$ & $0.29541(15)$ & $0.38931(4)$ & $0.0266(3)$ \\
\hline $\mathrm{O} 4$ & $0.26028(4)$ & $0.55159(15)$ & $0.35018(3)$ & $0.0244(3)$ \\
\hline $\mathrm{C} 1$ & $0.16537(6)$ & $0.6161(2)$ & $0.57029(5)$ & $0.0201(3)$ \\
\hline $\mathrm{C} 2$ & $0.21299(6)$ & $0.6304(2)$ & $0.59036(5)$ & 0.0198 \\
\hline $\mathrm{C} 3$ & $0.25165(6)$ & $0.5938(2)$ & $0.55724(5)$ & $0.0195(3)$ \\
\hline H3 & 0.2835 & 0.5432 & 0.5717 & $0.023 *$ \\
\hline C3A & $0.22152(6)$ & $0.4638(2)$ & $0.52660(5)$ & 0.0195 (3) \\
\hline $\mathrm{C} 3 \mathrm{~B}$ & $0.23500(6)$ & $0.4674(2)$ & $0.47966(5)$ & $0.0197(3)$ \\
\hline $\mathrm{C} 4$ & $0.26706(6)$ & $0.3619(2)$ & $0.45845(5)$ & $0.0210(4)$ \\
\hline $\mathrm{H} 4$ & 0.2842 & 0.2711 & 0.4736 & $0.025 *$ \\
\hline $\mathrm{C} 5$ & $0.27349(6)$ & $0.3925(2)$ & $0.41453(5)$ & $0.0210(4)$ \\
\hline C6 & $0.24940(6)$ & $0.5322(2)$ & $0.39281(5)$ & $0.0206(4)$ \\
\hline $\mathrm{C} 7$ & $0.21706(6)$ & $0.6363(2)$ & $0.41435(5)$ & $0.0218(4)$ \\
\hline $\mathrm{H7}$ & 0.2006 & 0.7297 & 0.3998 & $0.026 *$ \\
\hline C7A & $0.20952(6)$ & $0.6002(2)$ & $0.45771(5)$ & $0.0196(3)$ \\
\hline $\mathrm{C} 8$ & $0.17978(6)$ & $0.6978(2)$ & $0.48975(5)$ & 0.0205 (4) \\
\hline H8 & 0.1478 & 0.7487 & 0.4756 & $0.025^{*}$ \\
\hline C8A & $0.16948(6)$ & $0.5569(2)$ & $0.52346(5)$ & $0.0201(3)$ \\
\hline H8A & 0.1407 & 0.4817 & 0.5127 & $0.024 *$ \\
\hline $\mathrm{C} 9$ & $0.21579(6)$ & $0.8298(2)$ & $0.51258(5)$ & $0.0207(3)$ \\
\hline H9A & 0.2247 & 0.9154 & 0.4911 & $0.025 *$ \\
\hline H9B & 0.1985 & 0.8878 & 0.5357 & $0.025 *$ \\
\hline N10 & $0.26251(5)$ & $0.74768(17)$ & $0.53217(4)$ & $0.0200(3)$ \\
\hline $\mathrm{C} 11$ & $0.11459(6)$ & $0.6438(2)$ & $0.58631(5)$ & $0.0205(3)$ \\
\hline $\mathrm{C} 12$ & $0.10117(7)$ & $0.5756(2)$ & $0.62547(6)$ & $0.0291(4)$ \\
\hline
\end{tabular}




\begin{tabular}{|c|c|c|c|c|}
\hline H12 & 0.1255 & 0.5114 & 0.6431 & $0.035^{*}$ \\
\hline $\mathrm{C} 13$ & $0.05300(7)$ & $0.5997(2)$ & $0.63919(6)$ & $0.0330(4)$ \\
\hline H13 & 0.0445 & 0.5506 & 0.6658 & $0.040^{*}$ \\
\hline $\mathrm{C} 14$ & $0.01719(6)$ & $0.6951(2)$ & $0.61416(6)$ & $0.0294(4)$ \\
\hline H14 & -0.0156 & 0.7138 & 0.6237 & $0.035^{*}$ \\
\hline $\mathrm{C} 15$ & $0.03000(6)$ & $0.7628(2)$ & $0.57498(6)$ & $0.0286(4)$ \\
\hline H15 & 0.0058 & 0.8286 & 0.5577 & $0.034 *$ \\
\hline $\mathrm{C} 16$ & $0.07783(6)$ & $0.7357(2)$ & $0.56054(5)$ & $0.0248(4)$ \\
\hline H16 & 0.0856 & 0.7797 & 0.5331 & $0.030^{*}$ \\
\hline $\mathrm{C} 17$ & $0.23166(6)$ & $0.6887(2)$ & $0.63484(5)$ & $0.0216(4)$ \\
\hline $\mathrm{C} 18$ & $0.21198(7)$ & $0.8065(3)$ & $0.70192(5)$ & $0.0349(5)$ \\
\hline H18A & 0.2401 & 0.8868 & 0.7012 & $0.052 *$ \\
\hline H18B & 0.2235 & 0.7076 & 0.7192 & $0.052 *$ \\
\hline $\mathrm{H} 18 \mathrm{C}$ & 0.1837 & 0.8602 & 0.7152 & $0.052 *$ \\
\hline C19 & $0.22182(6)$ & $0.2883(2)$ & $0.54690(5)$ & $0.0243(4)$ \\
\hline H19A & 0.1990 & 0.2142 & 0.5287 & $0.036^{*}$ \\
\hline H19B & 0.2103 & 0.2954 & 0.5763 & $0.036^{*}$ \\
\hline H19C & 0.2564 & 0.2424 & 0.5487 & $0.036^{*}$ \\
\hline $\mathrm{C} 20$ & $0.24521(7)$ & $0.7058(2)$ & $0.32898(5)$ & $0.0300(4)$ \\
\hline $\mathrm{H} 20 \mathrm{~A}$ & 0.2585 & 0.7100 & 0.3002 & $0.045^{*}$ \\
\hline $\mathrm{H} 20 \mathrm{~B}$ & 0.2588 & 0.8010 & 0.3467 & $0.045^{*}$ \\
\hline $\mathrm{H} 20 \mathrm{C}$ & 0.2080 & 0.7124 & 0.3254 & $0.045^{*}$ \\
\hline $\mathrm{C} 21$ & $0.32021(7)$ & $0.1375(2)$ & $0.40771(6)$ & $0.0308(4)$ \\
\hline $\mathrm{H} 21 \mathrm{~A}$ & 0.3390 & 0.0767 & 0.3865 & $0.046^{*}$ \\
\hline $\mathrm{H} 21 \mathrm{~B}$ & 0.2911 & 0.0697 & 0.4149 & $0.046^{*}$ \\
\hline $\mathrm{H} 21 \mathrm{C}$ & 0.3427 & 0.1583 & 0.4343 & $0.046^{*}$ \\
\hline $\mathrm{C} 22$ & $0.29463(6)$ & $0.8685(2)$ & $0.55748(5)$ & $0.0239(4)$ \\
\hline $\mathrm{H} 22 \mathrm{~A}$ & 0.2753 & 0.9199 & 0.5798 & $0.036^{*}$ \\
\hline $\mathrm{H} 22 \mathrm{~B}$ & 0.3057 & 0.9564 & 0.5380 & $0.036^{*}$ \\
\hline $\mathrm{H} 22 \mathrm{C}$ & 0.3245 & 0.8103 & 0.5716 & $0.036^{*}$ \\
\hline O5 & $0.04526(4)$ & $0.27952(16)$ & $0.18932(4)$ & $0.0306(3)$ \\
\hline O6 & $0.12346(4)$ & $0.37557(16)$ & $0.17935(4)$ & $0.0302(3)$ \\
\hline O7 & $0.04963(4)$ & $-0.16000(15)$ & $0.44959(4)$ & 0.0258 \\
\hline O8 & $0.11516(4)$ & $0.06677(16)$ & $0.48935(3)$ & 0.0255 \\
\hline $\mathrm{C} 23$ & $0.16239(6)$ & $0.1905(2)$ & $0.25797(5)$ & 0.0188 \\
\hline $\mathrm{C} 24$ & $0.11291(6)$ & $0.2176(2)$ & $0.24310(5)$ & $0.0185(3)$ \\
\hline $\mathrm{C} 25$ & $0.07877(6)$ & $0.1781(2)$ & $0.27969(5)$ & $0.0185(3)$ \\
\hline $\mathrm{H} 25$ & 0.0444 & 0.1382 & 0.2677 & $0.022 *$ \\
\hline $\mathrm{C} 25 \mathrm{~A}$ & $0.10949(6)$ & $0.0350(2)$ & $0.30487(5)$ & $0.0193(3)$ \\
\hline $\mathrm{C} 25 \mathrm{~B}$ & $0.10346(6)$ & $0.0281(2)$ & $0.35363(5)$ & $0.0192(3)$ \\
\hline $\mathrm{C} 26$ & $0.07316(6)$ & $-0.0761(2)$ & $0.37696(5)$ & $0.0204(3)$ \\
\hline $\mathrm{H} 26$ & 0.0508 & -0.1554 & 0.3623 & $0.024 *$ \\
\hline $\mathrm{C} 27$ & $0.07641(6)$ & $-0.0616(2)$ & $0.42257(5)$ & $0.0214(4)$ \\
\hline $\mathrm{C} 28$ & $0.10869(6)$ & $0.0601(2)$ & $0.44392(5)$ & $0.0217(4)$ \\
\hline $\mathrm{C} 29$ & $0.13786(6)$ & $0.1658(2)$ & $0.42003(5)$ & $0.0219(4)$ \\
\hline $\mathrm{H} 29$ & 0.1591 & 0.2493 & 0.4343 & $0.026^{*}$ \\
\hline $\mathrm{C} 29 \mathrm{~A}$ & $0.13541(6)$ & $0.1474(2)$ & $0.37498(5)$ & $0.0205(3)$ \\
\hline $\mathrm{C} 30$ & $0.16122(6)$ & $0.2481(2)$ & $0.34132(5)$ & $0.0204(4)$ \\
\hline
\end{tabular}




\begin{tabular}{|c|c|c|c|c|}
\hline $\mathrm{H} 30$ & 0.1958 & 0.2889 & 0.3526 & $0.025^{*}$ \\
\hline $\mathrm{C} 30 \mathrm{~A}$ & $0.16310(6)$ & $0.1158(2)$ & $0.30369(5)$ & $0.0198(3)$ \\
\hline $\mathrm{H} 30 \mathrm{~A}$ & 0.1911 & 0.0318 & 0.3098 & $0.024 *$ \\
\hline $\mathrm{C} 31$ & $0.12553(6)$ & $0.3929(2)$ & $0.32492(5)$ & $0.0210(4)$ \\
\hline H31A & 0.1217 & 0.4732 & 0.3491 & $0.025 *$ \\
\hline H31B & 0.1406 & 0.4545 & 0.3012 & $0.025^{*}$ \\
\hline N32 & $0.07522(5)$ & $0.32672(17)$ & $0.30861(4)$ & $0.0199(3)$ \\
\hline $\mathrm{C} 33$ & $0.21087(6)$ & $0.2266(2)$ & $0.23888(5)$ & $0.0201(3)$ \\
\hline C34 & $0.21906(6)$ & $0.1952(2)$ & $0.19520(5)$ & 0.0259 \\
\hline $\mathrm{H} 34$ & 0.1926 & 0.1476 & 0.1761 & $0.031^{*}$ \\
\hline $\mathrm{C} 35$ & $0.26551(7)$ & $0.2328(2)$ & $0.17949(6)$ & $0.0310(4)$ \\
\hline $\mathrm{H} 35$ & 0.2705 & 0.2098 & 0.1498 & $0.037^{*}$ \\
\hline $\mathrm{C} 36$ & $0.30473(7)$ & $0.3035(2)$ & $0.20663(6)$ & $0.0293(4)$ \\
\hline H36 & 0.3362 & 0.3308 & 0.1956 & $0.035^{*}$ \\
\hline $\mathrm{C} 37$ & $0.29726(6)$ & $0.3338(2)$ & $0.25022(6)$ & $0.0279(4)$ \\
\hline $\mathrm{H} 37$ & 0.3237 & 0.3829 & 0.2691 & $0.033^{*}$ \\
\hline $\mathrm{C} 38$ & $0.25138(6)$ & $0.2928(2)$ & $0.26632(5)$ & 0.0235 \\
\hline $\mathrm{H} 38$ & 0.2473 & 0.3097 & 0.2964 & $0.028^{*}$ \\
\hline C39 & $0.09013(6)$ & $0.2923(2)$ & $0.20193(5)$ & $0.0209(4)$ \\
\hline $\mathrm{C} 40$ & $0.10380(8)$ & $0.4429(3)$ & $0.13759(6)$ & $0.0380(5)$ \\
\hline $\mathrm{H} 40 \mathrm{~A}$ & 0.0890 & 0.3515 & 0.1191 & $0.057^{*}$ \\
\hline $\mathrm{H} 40 \mathrm{~B}$ & 0.1315 & 0.4960 & 0.1233 & $0.057^{*}$ \\
\hline $\mathrm{H} 40 \mathrm{C}$ & 0.0776 & 0.5272 & 0.1420 & $0.057^{*}$ \\
\hline $\mathrm{C} 41$ & $0.10082(6)$ & $-0.1333(2)$ & $0.28122(5)$ & $0.0230(4)$ \\
\hline $\mathrm{H} 41 \mathrm{~A}$ & 0.1229 & -0.2193 & 0.2958 & $0.035^{*}$ \\
\hline H41B & 0.1088 & -0.1213 & 0.2508 & $0.035^{*}$ \\
\hline $\mathrm{H} 41 \mathrm{C}$ & 0.0652 & -0.1672 & 0.2819 & $0.035^{*}$ \\
\hline $\mathrm{C} 42$ & $0.07612(7)$ & $0.1543(2)$ & $0.50948(5)$ & $0.0289(4)$ \\
\hline $\mathrm{H} 42 \mathrm{~A}$ & 0.0696 & 0.2630 & 0.4949 & $0.043^{*}$ \\
\hline $\mathrm{H} 42 \mathrm{~B}$ & 0.0870 & 0.1734 & 0.5404 & $0.043^{*}$ \\
\hline $\mathrm{H} 42 \mathrm{C}$ & 0.0449 & 0.0867 & 0.5070 & $0.043^{*}$ \\
\hline $\mathrm{C} 43$ & $0.02597(7)$ & $-0.3088(2)$ & $0.43054(6)$ & $0.0338(4)$ \\
\hline $\mathrm{H} 43 \mathrm{~A}$ & -0.0009 & -0.2763 & 0.4080 & $0.051^{*}$ \\
\hline H43B & 0.0112 & -0.3745 & 0.4533 & $0.051^{*}$ \\
\hline $\mathrm{H} 43 \mathrm{C}$ & 0.0515 & -0.3771 & 0.4173 & $0.051^{*}$ \\
\hline $\mathrm{C} 44$ & $0.04325(6)$ & $0.4598(2)$ & $0.28805(5)$ & $0.0245(4)$ \\
\hline $\mathrm{H} 44 \mathrm{~A}$ & 0.0379 & 0.5475 & 0.3096 & $0.037^{*}$ \\
\hline H44B & 0.0104 & 0.4122 & 0.2769 & $0.037^{*}$ \\
\hline $\mathrm{H} 44 \mathrm{C}$ & 0.0600 & 0.5089 & 0.2638 & $0.037^{*}$ \\
\hline O9 & $0.38416(4)$ & $0.20757(16)$ & $0.51668(4)$ & $0.0281(3)$ \\
\hline $\mathrm{O} 10$ & $0.46237(4)$ & $0.31084(16)$ & $0.50926(4)$ & $0.0283(3)$ \\
\hline O11 & $0.36852(5)$ & $-0.25476(16)$ & $0.76825(4)$ & 0.0337 \\
\hline $\mathrm{O} 12$ & $0.40905(5)$ & $-0.00499(16)$ & $0.81049(4)$ & $0.0346(3)$ \\
\hline $\mathrm{C} 45$ & $0.49830(6)$ & $0.1339(2)$ & $0.59067(5)$ & 0.0194 \\
\hline $\mathrm{C} 46$ & $0.44971(6)$ & $0.1511(2)$ & $0.57266(5)$ & $0.0191(3)$ \\
\hline C47 & $0.41323(6)$ & $0.0987(2)$ & $0.60641(5)$ & $0.0204(4)$ \\
\hline $\mathrm{H} 47$ & 0.3809 & 0.0510 & 0.5920 & $0.025^{*}$ \\
\hline C47A & $0.44527(6)$ & -0.0399 & $0.63274(5)$ & 0.0208 \\
\hline
\end{tabular}




\begin{tabular}{|c|c|c|c|c|}
\hline C47B & $0.43428(6)$ & $-0.0539(2)$ & $0.68019(5)$ & 0.0217 (4) \\
\hline C48 & $0.40304(6)$ & -0.1680 & $0.70002(5)$ & $0.0246(4)$ \\
\hline $\mathrm{H} 48$ & 0.3862 & -0.2562 & 0.6836 & $0.030^{*}$ \\
\hline C49 & $0.39714(6)$ & $-0.1497(2)$ & $0.74438(5)$ & $0.0261(4)$ \\
\hline C50 & $0.42073(7)$ & $-0.0153(2)$ & $0.76786(5)$ & 0.0274 \\
\hline C51 & $0.45268(6)$ & $0.0966(2)$ & $0.74807(5)$ & $0.0263(4)$ \\
\hline H51 & 0.4691 & 0.1863 & 0.7642 & $0.032 *$ \\
\hline C51A & $0.45971(6)$ & $0.0730(2)$ & $0.70407(5)$ & 0.0233 \\
\hline C52 & $0.48757(6)$ & $0.1839(2)$ & $0.67344(5)$ & $0.0221(4)$ \\
\hline H52 & 0.5199 & 0.2318 & 0.6878 & $0.027^{*}$ \\
\hline C52A & $0.49672(6)$ & $0.0566(2)$ & $0.63598(5)$ & $0.0206(4)$ \\
\hline H52A & 0.5263 & -0.0194 & 0.6439 & $0.025^{*}$ \\
\hline C53 & $0.45036(6)$ & $0.3205(2)$ & $0.65498(5)$ & 0.0223 \\
\hline H53A & 0.4424 & 0.3980 & 0.6787 & $0.027^{*}$ \\
\hline H53B & 0.4662 & 0.3871 & 0.6326 & $0.027^{*}$ \\
\hline N54 & $0.40313(5)$ & $0.24188(18)$ & $0.63536(4)$ & 0.0219 \\
\hline C55 & $0.54798(6)$ & $0.1758(2)$ & $0.57438(5)$ & 0.0201 \\
\hline C56 & $0.55974(6)$ & $0.1444(2)$ & $0.53162(5)$ & $0.0235(4)$ \\
\hline H56 & 0.5348 & 0.0956 & 0.5112 & $0.028^{*}$ \\
\hline C57 & $0.60753(6)$ & $0.1839(2)$ & $0.51869(6)$ & 0.0273 \\
\hline H57 & 0.6151 & 0.1607 & 0.4896 & $0.033^{*}$ \\
\hline C58 & $0.64441(6)$ & $0.2571(2)$ & $0.54792(5)$ & $0.0258(4)$ \\
\hline $\mathrm{H} 58$ & 0.6768 & 0.2855 & 0.5388 & $0.031 *$ \\
\hline C59 & $0.63324(6)$ & $0.2882(2)$ & $0.59062(5)$ & 0.0253 \\
\hline H59 & 0.6581 & 0.3390 & 0.6108 & $0.030^{*}$ \\
\hline C60 & $0.58591(6)$ & $0.2452(2)$ & $0.60395(5)$ & 0.0229 \\
\hline H60 & 0.5792 & 0.2630 & 0.6335 & $0.027 *$ \\
\hline C61 & $0.42839(6)$ & $0.2241(2)$ & $0.53060(5)$ & $0.0212(4)$ \\
\hline C62 & $0.44417(7)$ & 0.3744 & $0.46658(5)$ & 0.0337 (4) \\
\hline H62A & 0.4133 & 0.4410 & 0.4689 & $0.050^{*}$ \\
\hline H62B & 0.4366 & 0.2796 & 0.4466 & $0.050^{*}$ \\
\hline H62C & 0.4704 & 0.4457 & 0.4553 & $0.050^{*}$ \\
\hline C63 & $0.44378(7)$ & $-0.2058(2)$ & $0.60785(5)$ & $0.0260(4)$ \\
\hline H63A & 0.4673 & -0.2860 & 0.6231 & $0.039^{*}$ \\
\hline H63B & 0.4538 & -0.1864 & 0.5783 & $0.039^{*}$ \\
\hline H63C & 0.4092 & -0.2519 & 0.6061 & $0.039 *$ \\
\hline C64 & $0.42125(8)$ & $0.1456(3)$ & $0.83337(6)$ & $0.0406(5)$ \\
\hline H64A & 0.4076 & 0.1415 & 0.8620 & $0.061^{*}$ \\
\hline H64B & 0.4064 & 0.2415 & 0.8167 & $0.061 *$ \\
\hline H64C & 0.4583 & 0.1586 & 0.8374 & $0.061 *$ \\
\hline C65 & $0.34943(7)$ & $-0.4060(2)$ & $0.74740(6)$ & $0.0362(5)$ \\
\hline H65A & 0.3315 & -0.4725 & 0.7681 & $0.054 *$ \\
\hline H65B & 0.3778 & -0.4719 & 0.7378 & $0.054 *$ \\
\hline H65C & 0.3259 & -0.3770 & 0.7220 & $0.054 *$ \\
\hline C66 & $0.36905(6)$ & $0.3679(2)$ & $0.61390(5)$ & $0.0253(4)$ \\
\hline H66A & 0.3386 & 0.3120 & 0.6001 & $0.038^{*}$ \\
\hline H66B & 0.3865 & 0.4270 & 0.5915 & $0.038^{*}$ \\
\hline H66C & 0.3592 & 0.4492 & 0.6356 & $0.038^{*}$ \\
\hline
\end{tabular}


Atomic displacement parameters $\left(\AA^{2}\right)$

\begin{tabular}{|c|c|c|c|c|c|c|}
\hline & $U^{11}$ & $U^{22}$ & $U^{33}$ & $U^{12}$ & $U^{13}$ & $U^{23}$ \\
\hline O1 & $0.0238(6)$ & $0.0403(8)$ & $0.0228(6)$ & $0.0006(6)$ & $-0.0001(5)$ & $-0.0031(5)$ \\
\hline $\mathrm{O} 2$ & $0.0268(6)$ & $0.0402(8)$ & $0.0210(6)$ & $0.0067(6)$ & $0.0022(5)$ & $-0.0091(5)$ \\
\hline $\mathrm{O} 3$ & $0.0314(7)$ & $0.0264(7)$ & $0.0226(6)$ & $0.0071(5)$ & $0.0060(5)$ & $-0.0020(5)$ \\
\hline $\mathrm{O} 4$ & $0.0300(6)$ & $0.0262(7)$ & $0.0170(5)$ & $-0.0005(5)$ & 0.0025 & $0.0001(5)$ \\
\hline $\mathrm{C} 1$ & $0.0208(8)$ & $0.0184(9)$ & $0.0213(8)$ & $0.0004(7)$ & $0.0033(6)$ & $0.0016(7)$ \\
\hline $\mathrm{C} 2$ & $0.0204(8)$ & $0.0195(9)$ & $0.0196(8)$ & $-0.0001(7)$ & $0.0027(6)$ & $0.0007(7)$ \\
\hline $\mathrm{C} 3$ & $0.0189(8)$ & $0.0208(9)$ & $0.0186(7)$ & $0.0017(7)$ & $0.0013(6)$ & $0.0009(7)$ \\
\hline $\mathrm{C} 3 \mathrm{~A}$ & $0.0195(8)$ & $0.0193(9)$ & $0.0198(8)$ & $0.0003(7)$ & $0.0024(6)$ & $-0.0008(7)$ \\
\hline $\mathrm{C} 3 \mathrm{~B}$ & $0.0185(8)$ & $0.0196(9)$ & $0.0209(8)$ & $-0.0015(7)$ & $0.0012(6)$ & $-0.0021(7)$ \\
\hline $\mathrm{C} 4$ & $0.0198(8)$ & $0.0205(9)$ & $0.0224(8)$ & $-0.0007(7)$ & $0.0002(6)$ & $0.0004(7)$ \\
\hline $\mathrm{C} 5$ & $0.0191(8)$ & $0.0224(9)$ & $0.0216(8)$ & $-0.0004(7)$ & $0.0022(6)$ & $-0.0044(7)$ \\
\hline C6 & $0.0208(8)$ & $0.0242(9)$ & $0.0165(7)$ & $-0.0040(7)$ & $-0.0002(6)$ & $-0.0011(7)$ \\
\hline $\mathrm{C} 7$ & $0.0213(8)$ & $0.0218(9)$ & $0.0217(8)$ & $-0.0008(7)$ & $-0.0011(6)$ & $0.0003(7)$ \\
\hline C7A & $0.0177(8)$ & $0.0193(9)$ & $0.0215(8)$ & -0.0021 & $-0.0005(6)$ & $-0.0023(7)$ \\
\hline $\mathrm{C} 8$ & $0.0203(8)$ & $0.0228(9)$ & $0.0181(8)$ & $0.0033(7)$ & $0.0007(6)$ & $0.0005(7)$ \\
\hline C8A & $0.0174(8)$ & 0.0209 (9) & $0.0218(8)$ & $-0.0006(7)$ & $0.0014(6)$ & $-0.0018(7)$ \\
\hline C9 & $0.0211(8)$ & $0.0197(9)$ & $0.0214(8)$ & $0.0022(7)$ & $0.0028(6)$ & $0.0015(7)$ \\
\hline N10 & $0.0186(7)$ & $0.0199(7)$ & $0.0214(7)$ & $0.0002(6)$ & $0.0011(5)$ & $-0.0003(6)$ \\
\hline $\mathrm{C} 11$ & $0.0196(8)$ & $0.0214(9)$ & $0.0207(8)$ & $-0.0027(7)$ & $0.0025(6)$ & $-0.0013(7)$ \\
\hline $\mathrm{C} 12$ & $0.0264(9)$ & $0.0306(10)$ & $0.0308(9)$ & $0.0036(8)$ & $0.0061(7)$ & $0.0076(8)$ \\
\hline $\mathrm{C} 13$ & $0.0310(10)$ & $0.0354(11)$ & $0.0345(10)$ & $-0.0002(8)$ & $0.0146(8)$ & $0.0077(9)$ \\
\hline $\mathrm{C} 14$ & $0.0212(9)$ & $0.0313(11)$ & $0.0367(10)$ & $-0.0027(8)$ & $0.0085(7)$ & $-0.0046(8)$ \\
\hline $\mathrm{C} 15$ & $0.0205(9)$ & $0.0378(11)$ & $0.0271(9)$ & $0.0037(8)$ & $-0.0002(7)$ & $-0.0032(8)$ \\
\hline $\mathrm{C} 16$ & $0.0222(8)$ & $0.0329(10)$ & $0.0191(8)$ & $0.0027(8)$ & $0.0010(6)$ & $-0.0010(7)$ \\
\hline $\mathrm{C} 17$ & $0.0230(9)$ & $0.0224(9)$ & $0.0199(8)$ & $0.0005(7)$ & $0.0048(6)$ & $0.0016(7)$ \\
\hline C18 & $0.0402(11)$ & $0.0440(12)$ & $0.0203(9)$ & $0.0054(9)$ & $0.0020(7)$ & $-0.0089(8)$ \\
\hline C19 & $0.0274(9)$ & $0.0227(10)$ & $0.0227(8)$ & $-0.0006(7)$ & $0.0021(7)$ & $-0.0007(7)$ \\
\hline $\mathrm{C} 20$ & $0.0361(10)$ & $0.0325(11)$ & $0.0211(8)$ & $0.0020(8)$ & $0.0006(7)$ & $0.0061(8)$ \\
\hline $\mathrm{C} 21$ & $0.0351(10)$ & $0.0272(10)$ & $0.0308(9)$ & $0.0084(8)$ & $0.0067(8)$ & $-0.0024(8)$ \\
\hline $\mathrm{C} 22$ & $0.0208(8)$ & $0.0247(10)$ & $0.0260(8)$ & $-0.0038(7)$ & $0.0022(7)$ & $-0.0037(7)$ \\
\hline $\mathrm{O} 5$ & $0.0254(6)$ & $0.0397(8)$ & $0.0259(6)$ & $-0.0022(6)$ & $-0.0025(5)$ & $0.0053(6)$ \\
\hline O6 & $0.0285(7)$ & $0.0382(8)$ & $0.0235(6)$ & -0.0069 & 0.0000 & $0.0125(6)$ \\
\hline O7 & $0.0278(6)$ & $0.0281(7)$ & $0.0222(6)$ & -0.0059 (5) & $0.0058(5)$ & $0.0051(5)$ \\
\hline O8 & $0.0254(6)$ & $0.0354(7)$ & $0.0157(5)$ & $0.0021(5)$ & $0.0023(4)$ & $0.0013(5)$ \\
\hline $\mathrm{C} 23$ & $0.0211(8)$ & $0.0175(9)$ & $0.0181(7)$ & $-0.0006(7)$ & $0.0031(6)$ & $-0.0023(6)$ \\
\hline $\mathrm{C} 24$ & $0.0211(8)$ & $0.0176(9)$ & $0.0171(7)$ & $-0.0017(7)$ & $0.0027(6)$ & $-0.0006(6)$ \\
\hline $\mathrm{C} 25$ & $0.0192(8)$ & $0.0201(9)$ & $0.0164(7)$ & $-0.0016(7)$ & $0.0027(6)$ & $-0.0004(6)$ \\
\hline $\mathrm{C} 25 \mathrm{~A}$ & $0.0204(8)$ & $0.0205(9)$ & $0.0170(7)$ & $-0.0011(7)$ & $0.0011(6)$ & $0.0007(7)$ \\
\hline $\mathrm{C} 25 \mathrm{~B}$ & $0.0173(8)$ & $0.0200(9)$ & $0.0203(8)$ & $0.0006(7)$ & $0.0008(6)$ & $0.0011(7)$ \\
\hline $\mathrm{C} 26$ & $0.0194(8)$ & $0.0201(9)$ & $0.0213(8)$ & $-0.0006(7)$ & $0.0000(6)$ & $0.0020(7)$ \\
\hline $\mathrm{C} 27$ & $0.0206(8)$ & $0.0229(9)$ & $0.0211(8)$ & $0.0005(7)$ & $0.0032(6)$ & $0.0046(7)$ \\
\hline $\mathrm{C} 28$ & $0.0226(8)$ & $0.0269(10)$ & $0.0153(7)$ & $0.0024(7)$ & $0.0000(6)$ & $0.0026(7)$ \\
\hline $\mathrm{C} 29$ & $0.0205(8)$ & $0.0243(9)$ & $0.0206(8)$ & $-0.0027(7)$ & $0.0001(6)$ & $0.0004(7)$ \\
\hline C29A & $0.0194(8)$ & $0.0227(9)$ & $0.0191(8)$ & -0.0009 (7) & $0.0008(6)$ & $0.0021(7)$ \\
\hline $\mathrm{C} 30$ & $0.0193(8)$ & $0.0247(9)$ & $0.0172(7)$ & $-0.0050(7)$ & $0.0014(6)$ & $-0.0005(7)$ \\
\hline
\end{tabular}




\begin{tabular}{|c|c|c|c|c|c|c|}
\hline $\mathrm{C} 30 \mathrm{~A}$ & $0.0184(8)$ & $0.0221(9)$ & $0.0189(8)$ & $0.0006(7)$ & $0.0017(6)$ & $0.0005(7)$ \\
\hline C31 & $0.0242(8)$ & $0.0200(9)$ & $0.0191(8)$ & $-0.0040(7)$ & $0.0045(6)$ & $-0.0021(7)$ \\
\hline N32 & $0.0214(7)$ & $0.0184(7)$ & $0.0200(7)$ & $-0.0005(6)$ & $0.0026(5)$ & $-0.0018(6)$ \\
\hline C33 & $0.0201(8)$ & $0.0184(9)$ & $0.0223(8)$ & $0.0013(7)$ & $0.0041(6)$ & $0.0008(7)$ \\
\hline $\mathrm{C} 34$ & $0.0258(9)$ & $0.0305(10)$ & $0.0218(8)$ & $-0.0028(8)$ & $0.0046(7)$ & $-0.0058(7)$ \\
\hline $\mathrm{C} 35$ & $0.0302(10)$ & $0.0395(12)$ & $0.0248(9)$ & $-0.0016(9)$ & $0.0109(7)$ & $-0.0027(8)$ \\
\hline $\mathrm{C} 36$ & $0.0232(9)$ & $0.0323(11)$ & $0.0336(10)$ & $0.0014(8)$ & $0.0102(7)$ & $0.0021(8)$ \\
\hline C37 & $0.0206(9)$ & $0.0320(11)$ & $0.0314(9)$ & $-0.0012(8)$ & $0.0038(7)$ & $-0.0004(8)$ \\
\hline C38 & $0.0220(8)$ & $0.0291(10)$ & $0.0196(8)$ & $0.0000(7)$ & $0.0029(6)$ & -0.0003 \\
\hline C39 & $0.0223(9)$ & $0.0212(9)$ & $0.0196(8)$ & $-0.0020(7)$ & $0.0034(6)$ & $-0.0019(7)$ \\
\hline $\mathrm{C} 40$ & $0.0441(12)$ & $0.0455(13)$ & $0.0230(9)$ & $-0.0081(10)$ & $-0.0040(8)$ & $0.0134(9)$ \\
\hline $\mathrm{C} 41$ & $0.0272(9)$ & $0.0196(9)$ & $0.0218(8)$ & $-0.0012(7)$ & $-0.0005(7)$ & $-0.0006(7)$ \\
\hline $\mathrm{C} 42$ & $0.0282(9)$ & $0.0388(11)$ & $0.0202(8)$ & $0.0005(8)$ & $0.0043(7)$ & $-0.0016(8)$ \\
\hline $\mathrm{C} 43$ & $0.0391(11)$ & $0.0301(11)$ & $0.0335(10)$ & $-0.0092(9)$ & $0.0108(8)$ & $0.0021(8)$ \\
\hline $\mathrm{C} 44$ & $0.0253(9)$ & $0.0226(9)$ & $0.0264(8)$ & $0.0021(7)$ & $0.0068(7)$ & $0.0019(7)$ \\
\hline O9 & $0.0229(6)$ & $0.0366(8)$ & $0.0243(6)$ & $-0.0016(5)$ & $-0.0010(5)$ & $0.0026(5)$ \\
\hline $\mathrm{O} 10$ & $0.0267(6)$ & $0.0341(7)$ & $0.0238(6)$ & $-0.0040(5)$ & $0.0006(5)$ & $0.0099(5)$ \\
\hline O11 & $0.0405(7)$ & $0.0343(8)$ & $0.0281(6)$ & $-0.0021(6)$ & $0.0128(5)$ & $0.0076(6)$ \\
\hline $\mathrm{O} 12$ & $0.0493(8)$ & $0.0340(8)$ & $0.0212(6)$ & $-0.0002(6)$ & $0.0071(5)$ & $0.0008(6)$ \\
\hline $\mathrm{C} 45$ & $0.0222(8)$ & $0.0170(9)$ & $0.0194(8)$ & $-0.0006(7)$ & $0.0046(6)$ & $-0.0021(7)$ \\
\hline $\mathrm{C} 46$ & $0.0197(8)$ & $0.0188(9)$ & $0.0189(8)$ & $-0.0016(7)$ & $0.0029(6)$ & $-0.0015(7)$ \\
\hline $\mathrm{C} 47$ & $0.0184(8)$ & $0.0237(9)$ & $0.0193(8)$ & $-0.0013(7)$ & $0.0018(6)$ & $0.0008(7)$ \\
\hline C47A & $0.0198(8)$ & $0.0215(9)$ & $0.0211(8)$ & $-0.0003(7)$ & $0.0021(6)$ & $0.0016(7)$ \\
\hline C47B & $0.0193(8)$ & $0.0239(9)$ & $0.0222(8)$ & $0.0020(7)$ & $0.0030(6)$ & $0.0035(7)$ \\
\hline $\mathrm{C} 48$ & $0.0236(9)$ & $0.0256(10)$ & $0.0248(8)$ & $0.0019(7)$ & $0.0027(7)$ & $0.0046(7)$ \\
\hline $\mathrm{C} 49$ & $0.0271(9)$ & $0.0263(10)$ & $0.0256(9)$ & $0.0031(8)$ & $0.0063(7)$ & $0.0072(8)$ \\
\hline $\mathrm{C} 50$ & $0.0324(10)$ & $0.0320(11)$ & $0.0184(8)$ & $0.0078(8)$ & $0.0056(7)$ & $0.0069(7)$ \\
\hline $\mathrm{C} 51$ & $0.0293(9)$ & $0.0275(10)$ & $0.0218(8)$ & $0.0036(8)$ & $0.0008(7)$ & $0.0009(7)$ \\
\hline C51A & $0.0227(8)$ & $0.0268(10)$ & $0.0203(8)$ & $0.0040(7)$ & $0.0011(6)$ & $0.0039(7)$ \\
\hline $\mathrm{C} 52$ & $0.0227(8)$ & $0.0256(10)$ & $0.0181(8)$ & $-0.0027(7)$ & $0.0012(6)$ & $-0.0017(7)$ \\
\hline $\mathrm{C} 52 \mathrm{~A}$ & $0.0179(8)$ & $0.0216(9)$ & $0.0225(8)$ & $0.0013(7)$ & $0.0021(6)$ & $0.0016(7)$ \\
\hline $\mathrm{C} 53$ & $0.0246(9)$ & $0.0217(9)$ & $0.0207(8)$ & $-0.0016(7)$ & $0.0034(6)$ & $-0.0013(7)$ \\
\hline N54 & $0.0204(7)$ & $0.0236(8)$ & 0.0219 (7) & 0.0017 (6) & $0.0033(5)$ & $-0.0003(6)$ \\
\hline $\mathrm{C} 55$ & $0.0194(8)$ & $0.0185(9)$ & $0.0227(8)$ & $0.0018(7)$ & $0.0031(6)$ & $0.0004(7)$ \\
\hline $\mathrm{C} 56$ & $0.0220(8)$ & $0.0260(10)$ & $0.0232(8)$ & $-0.0024(7)$ & $0.0046(6)$ & $-0.0043(7)$ \\
\hline $\mathrm{C} 57$ & $0.0274(9)$ & $0.0304(10)$ & $0.0252(9)$ & $0.0001(8)$ & $0.0087(7)$ & $-0.0013(8)$ \\
\hline $\mathrm{C} 58$ & $0.0208(8)$ & $0.0274(10)$ & $0.0303(9)$ & $-0.0008(7)$ & $0.0074(7)$ & $0.0010(8)$ \\
\hline $\mathrm{C} 59$ & $0.0198(8)$ & $0.0287(10)$ & $0.0273(9)$ & $-0.0023(7)$ & 0.0009 (7) & $0.0020(8)$ \\
\hline $\mathrm{C} 60$ & $0.0226(8)$ & $0.0253(9)$ & $0.0211(8)$ & $0.0010(7)$ & $0.0031(6)$ & $0.0004(7)$ \\
\hline C61 & $0.0232(9)$ & $0.0209(9)$ & $0.0199(8)$ & $-0.0001(7)$ & $0.0045(6)$ & $-0.0018(7)$ \\
\hline C62 & $0.0393(11)$ & $0.0379(12)$ & $0.0235(9)$ & $-0.0027(9)$ & $0.0012(8)$ & $0.0098(8)$ \\
\hline C63 & $0.0274(9)$ & $0.0228(10)$ & $0.0277(9)$ & $-0.0012(7)$ & $0.0018(7)$ & $0.0016(7)$ \\
\hline C64 & $0.0562(13)$ & $0.0413(13)$ & $0.0230(9)$ & $-0.0053(10)$ & $-0.0031(9)$ & $-0.0061(9)$ \\
\hline $\mathrm{C} 65$ & $0.0394(11)$ & $0.0330(11)$ & $0.0373(11)$ & $-0.0078(9)$ & $0.0088(8)$ & $0.0058(9)$ \\
\hline C66 & $0.0251(9)$ & $0.0247(10)$ & $0.0267(9)$ & $0.0044(7)$ & $0.0057(7)$ & $0.0026(7)$ \\
\hline
\end{tabular}


Geometric parameters $\left(\AA,{ }^{\circ}\right)$

\begin{tabular}{|c|c|c|c|}
\hline $\mathrm{O} 1-\mathrm{C} 17$ & $1.2105(19)$ & $\mathrm{C} 31-\mathrm{N} 32$ & $1.474(2)$ \\
\hline $\mathrm{O} 2-\mathrm{C} 17$ & $1.3477(19)$ & $\mathrm{C} 31-\mathrm{H} 31 \mathrm{~A}$ & 0.9900 \\
\hline $\mathrm{O} 2-\mathrm{C} 18$ & $1.447(2)$ & $\mathrm{C} 31-\mathrm{H} 31 \mathrm{~B}$ & 0.9900 \\
\hline $\mathrm{O} 3-\mathrm{C} 5$ & $1.3737(19)$ & $\mathrm{N} 32-\mathrm{C} 44$ & $1.459(2)$ \\
\hline $\mathrm{O} 3-\mathrm{C} 21$ & $1.435(2)$ & $\mathrm{C} 33-\mathrm{C} 34$ & $1.400(2)$ \\
\hline $\mathrm{O} 4-\mathrm{C} 6$ & $1.3741(18)$ & $\mathrm{C} 33-\mathrm{C} 38$ & $1.403(2)$ \\
\hline $\mathrm{O} 4-\mathrm{C} 20$ & $1.425(2)$ & C34-C35 & $1.390(2)$ \\
\hline $\mathrm{C} 1-\mathrm{C} 2$ & $1.355(2)$ & C34-H34 & 0.9500 \\
\hline $\mathrm{C} 1-\mathrm{C} 11$ & $1.485(2)$ & $\mathrm{C} 35-\mathrm{C} 36$ & $1.389(3)$ \\
\hline $\mathrm{C} 1-\mathrm{C} 8 \mathrm{~A}$ & $1.527(2)$ & C $35-\mathrm{H} 35$ & 0.9500 \\
\hline $\mathrm{C} 2-\mathrm{C} 17$ & $1.485(2)$ & $\mathrm{C} 36-\mathrm{C} 37$ & $1.392(2)$ \\
\hline $\mathrm{C} 2-\mathrm{C} 3$ & $1.532(2)$ & $\mathrm{C} 36-\mathrm{H} 36$ & 0.9500 \\
\hline $\mathrm{C} 3-\mathrm{N} 10$ & $1.485(2)$ & $\mathrm{C} 37-\mathrm{C} 38$ & $1.387(2)$ \\
\hline $\mathrm{C} 3-\mathrm{C} 3 \mathrm{~A}$ & $1.565(2)$ & $\mathrm{C} 37-\mathrm{H} 37$ & 0.9500 \\
\hline $\mathrm{C} 3-\mathrm{H} 3$ & 1.0000 & C38-H38 & 0.9500 \\
\hline $\mathrm{C} 3 \mathrm{~A}-\mathrm{C} 3 \mathrm{~B}$ & $1.516(2)$ & $\mathrm{C} 40-\mathrm{H} 40 \mathrm{~A}$ & 0.9800 \\
\hline $\mathrm{C} 3 \mathrm{~A}-\mathrm{C} 19$ & $1.525(2)$ & $\mathrm{C} 40-\mathrm{H} 40 \mathrm{~B}$ & 0.9800 \\
\hline $\mathrm{C} 3 \mathrm{~A}-\mathrm{C} 8 \mathrm{~A}$ & $1.555(2)$ & $\mathrm{C} 40-\mathrm{H} 40 \mathrm{C}$ & 0.9800 \\
\hline $\mathrm{C} 3 \mathrm{~B}-\mathrm{C} 7 \mathrm{~A}$ & $1.391(2)$ & $\mathrm{C} 41-\mathrm{H} 41 \mathrm{~A}$ & 0.9800 \\
\hline $\mathrm{C} 3 \mathrm{~B}-\mathrm{C} 4$ & $1.393(2)$ & $\mathrm{C} 41-\mathrm{H} 41 \mathrm{~B}$ & 0.9800 \\
\hline $\mathrm{C} 4-\mathrm{C} 5$ & $1.396(2)$ & $\mathrm{C} 41-\mathrm{H} 41 \mathrm{C}$ & 0.9800 \\
\hline $\mathrm{C} 4-\mathrm{H} 4$ & 0.9500 & $\mathrm{C} 42-\mathrm{H} 42 \mathrm{~A}$ & 0.9800 \\
\hline $\mathrm{C} 5-\mathrm{C} 6$ & $1.415(2)$ & $\mathrm{C} 42-\mathrm{H} 42 \mathrm{~B}$ & 0.9800 \\
\hline $\mathrm{C} 6-\mathrm{C} 7$ & $1.396(2)$ & $\mathrm{C} 42-\mathrm{H} 42 \mathrm{C}$ & 0.9800 \\
\hline $\mathrm{C} 7-\mathrm{C} 7 \mathrm{~A}$ & $1.394(2)$ & $\mathrm{C} 43-\mathrm{H} 43 \mathrm{~A}$ & 0.9800 \\
\hline $\mathrm{C} 7-\mathrm{H} 7$ & 0.9500 & $\mathrm{C} 43-\mathrm{H} 43 \mathrm{~B}$ & 0.9800 \\
\hline $\mathrm{C} 7 \mathrm{~A}-\mathrm{C} 8$ & $1.524(2)$ & $\mathrm{C} 43-\mathrm{H} 43 \mathrm{C}$ & 0.9800 \\
\hline $\mathrm{C} 8-\mathrm{C} 9$ & $1.541(2)$ & $\mathrm{C} 44-\mathrm{H} 44 \mathrm{~A}$ & 0.9800 \\
\hline $\mathrm{C} 8-\mathrm{C} 8 \mathrm{~A}$ & $1.563(2)$ & $\mathrm{C} 44-\mathrm{H} 44 \mathrm{~B}$ & 0.9800 \\
\hline $\mathrm{C} 8-\mathrm{H} 8$ & 1.0000 & $\mathrm{C} 44-\mathrm{H} 44 \mathrm{C}$ & 0.9800 \\
\hline $\mathrm{C} 8 \mathrm{~A}-\mathrm{H} 8 \mathrm{~A}$ & 1.0000 & O9- C61 & $1.2148(19)$ \\
\hline $\mathrm{C} 9-\mathrm{N} 10$ & $1.475(2)$ & $\mathrm{O} 10-\mathrm{C} 61$ & $1.346(2)$ \\
\hline C9-H9A & 0.9900 & $\mathrm{O} 10-\mathrm{C} 62$ & $1.446(2)$ \\
\hline C9-H9B & 0.9900 & $\mathrm{O} 11-\mathrm{C} 49$ & $1.379(2)$ \\
\hline $\mathrm{N} 10-\mathrm{C} 22$ & $1.458(2)$ & $\mathrm{O} 11-\mathrm{C} 65$ & $1.430(2)$ \\
\hline $\mathrm{C} 11-\mathrm{C} 12$ & $1.393(2)$ & $\mathrm{O} 12-\mathrm{C} 50$ & 1.3744 (19) \\
\hline $\mathrm{C} 11-\mathrm{C} 16$ & $1.401(2)$ & $\mathrm{O} 12-\mathrm{C} 64$ & $1.409(2)$ \\
\hline $\mathrm{C} 12-\mathrm{C} 13$ & $1.388(2)$ & $\mathrm{C} 45-\mathrm{C} 46$ & $1.358(2)$ \\
\hline $\mathrm{C} 12-\mathrm{H} 12$ & 0.9500 & $\mathrm{C} 45-\mathrm{C} 55$ & $1.483(2)$ \\
\hline $\mathrm{C} 13-\mathrm{C} 14$ & $1.389(3)$ & $\mathrm{C} 45-\mathrm{C} 52 \mathrm{~A}$ & $1.525(2)$ \\
\hline $\mathrm{C} 13-\mathrm{H} 13$ & 0.9500 & $\mathrm{C} 46-\mathrm{C} 61$ & $1.481(2)$ \\
\hline $\mathrm{C} 14-\mathrm{C} 15$ & $1.387(2)$ & $\mathrm{C} 46-\mathrm{C} 47$ & $1.534(2)$ \\
\hline $\mathrm{C} 14-\mathrm{H} 14$ & 0.9500 & $\mathrm{C} 47-\mathrm{N} 54$ & $1.481(2)$ \\
\hline $\mathrm{C} 15-\mathrm{C} 16$ & $1.392(2)$ & $\mathrm{C} 47-\mathrm{C} 47 \mathrm{~A}$ & $1.567(2)$ \\
\hline $\mathrm{C} 15-\mathrm{H} 15$ & 0.9500 & $\mathrm{C} 47-\mathrm{H} 47$ & 1.0000 \\
\hline C16-H16 & 0.9500 & $\mathrm{C} 47 \mathrm{~A}-\mathrm{C} 47 \mathrm{~B}$ & $1.515(2)$ \\
\hline
\end{tabular}




\begin{tabular}{|c|c|c|c|}
\hline $\mathrm{C} 18-\mathrm{H} 18 \mathrm{~A}$ & 0.9800 & $\mathrm{C} 47 \mathrm{~A}-\mathrm{C} 63$ & $1.520(2)$ \\
\hline C18-H18B & 0.9800 & $\mathrm{C} 47 \mathrm{~A}-\mathrm{C} 52 \mathrm{~A}$ & $1.555(2)$ \\
\hline $\mathrm{C} 18-\mathrm{H} 18 \mathrm{C}$ & 0.9800 & $\mathrm{C} 47 \mathrm{~B}-\mathrm{C} 51 \mathrm{~A}$ & $1.383(2)$ \\
\hline C19-H19A & 0.9800 & $\mathrm{C} 47 \mathrm{~B}-\mathrm{C} 48$ & $1.400(2)$ \\
\hline C19-H19B & 0.9800 & $\mathrm{C} 48-\mathrm{C} 49$ & $1.393(2)$ \\
\hline C19-H19C & 0.9800 & $\mathrm{C} 48-\mathrm{H} 48$ & 0.9500 \\
\hline $\mathrm{C} 20-\mathrm{H} 20 \mathrm{~A}$ & 0.9800 & $\mathrm{C} 49-\mathrm{C} 50$ & $1.402(3)$ \\
\hline $\mathrm{C} 20-\mathrm{H} 20 \mathrm{~B}$ & 0.9800 & $\mathrm{C} 50-\mathrm{C} 51$ & $1.400(3)$ \\
\hline $\mathrm{C} 20-\mathrm{H} 20 \mathrm{C}$ & 0.9800 & $\mathrm{C} 51-\mathrm{C} 51 \mathrm{~A}$ & $1.393(2)$ \\
\hline $\mathrm{C} 21-\mathrm{H} 21 \mathrm{~A}$ & 0.9800 & C51-H51 & 0.9500 \\
\hline $\mathrm{C} 21-\mathrm{H} 21 \mathrm{~B}$ & 0.9800 & $\mathrm{C} 51 \mathrm{~A}-\mathrm{C} 52$ & $1.524(2)$ \\
\hline $\mathrm{C} 21-\mathrm{H} 21 \mathrm{C}$ & 0.9800 & $\mathrm{C} 52-\mathrm{C} 53$ & $1.537(2)$ \\
\hline $\mathrm{C} 22-\mathrm{H} 22 \mathrm{~A}$ & 0.9800 & $\mathrm{C} 52-\mathrm{C} 52 \mathrm{~A}$ & $1.566(2)$ \\
\hline $\mathrm{C} 22-\mathrm{H} 22 \mathrm{~B}$ & 0.9800 & $\mathrm{C} 52-\mathrm{H} 52$ & 1.0000 \\
\hline $\mathrm{C} 22-\mathrm{H} 22 \mathrm{C}$ & 0.9800 & $\mathrm{C} 52 \mathrm{~A}-\mathrm{H} 52 \mathrm{~A}$ & 1.0000 \\
\hline $\mathrm{O} 5-\mathrm{C} 39$ & $1.2177(19)$ & $\mathrm{C} 53-\mathrm{N} 54$ & $1.474(2)$ \\
\hline O6-C39 & $1.3414(19)$ & $\mathrm{C} 53-\mathrm{H} 53 \mathrm{~A}$ & 0.9900 \\
\hline $\mathrm{O} 6-\mathrm{C} 40$ & $1.443(2)$ & C53-H53B & 0.9900 \\
\hline $\mathrm{O} 7-\mathrm{C} 27$ & $1.3789(19)$ & N54-C66 & $1.462(2)$ \\
\hline $\mathrm{O} 7-\mathrm{C} 43$ & $1.435(2)$ & $\mathrm{C} 55-\mathrm{C} 56$ & 1.399 (2) \\
\hline $\mathrm{O} 8-\mathrm{C} 28$ & $1.3919(18)$ & $\mathrm{C} 55-\mathrm{C} 60$ & $1.403(2)$ \\
\hline $\mathrm{O} 8-\mathrm{C} 42$ & $1.428(2)$ & $\mathrm{C} 56-\mathrm{C} 57$ & $1.392(2)$ \\
\hline $\mathrm{C} 23-\mathrm{C} 24$ & $1.362(2)$ & $\mathrm{C} 56-\mathrm{H} 56$ & 0.9500 \\
\hline $\mathrm{C} 23-\mathrm{C} 33$ & $1.483(2)$ & $\mathrm{C} 57-\mathrm{C} 58$ & $1.391(2)$ \\
\hline $\mathrm{C} 23-\mathrm{C} 30 \mathrm{~A}$ & $1.523(2)$ & C57-H57 & 0.9500 \\
\hline $\mathrm{C} 24-\mathrm{C} 39$ & $1.476(2)$ & $\mathrm{C} 58-\mathrm{C} 59$ & $1.392(2)$ \\
\hline $\mathrm{C} 24-\mathrm{C} 25$ & $1.534(2)$ & $\mathrm{C} 58-\mathrm{H} 58$ & 0.9500 \\
\hline $\mathrm{C} 25-\mathrm{N} 32$ & $1.484(2)$ & $\mathrm{C} 59-\mathrm{C} 60$ & $1.392(2)$ \\
\hline $\mathrm{C} 25-\mathrm{C} 25 \mathrm{~A}$ & $1.560(2)$ & C59-H59 & 0.9500 \\
\hline $\mathrm{C} 25-\mathrm{H} 25$ & 1.0000 & $\mathrm{C} 60-\mathrm{H} 60$ & 0.9500 \\
\hline $\mathrm{C} 25 \mathrm{~A}-\mathrm{C} 25 \mathrm{~B}$ & $1.521(2)$ & $\mathrm{C} 62-\mathrm{H} 62 \mathrm{~A}$ & 0.9800 \\
\hline $\mathrm{C} 25 \mathrm{~A}-\mathrm{C} 41$ & $1.527(2)$ & C62-H62B & 0.9800 \\
\hline $\mathrm{C} 25 \mathrm{~A}-\mathrm{C} 30 \mathrm{~A}$ & 1.557 (2) & $\mathrm{C} 62-\mathrm{H} 62 \mathrm{C}$ & 0.9800 \\
\hline $\mathrm{C} 25 \mathrm{~B}-\mathrm{C} 26$ & $1.392(2)$ & $\mathrm{C} 63-\mathrm{H} 63 \mathrm{~A}$ & 0.9800 \\
\hline $\mathrm{C} 25 \mathrm{~B}-\mathrm{C} 29 \mathrm{~A}$ & $1.393(2)$ & $\mathrm{C} 63-\mathrm{H} 63 \mathrm{~B}$ & 0.9800 \\
\hline $\mathrm{C} 26-\mathrm{C} 27$ & $1.401(2)$ & $\mathrm{C} 63-\mathrm{H} 63 \mathrm{C}$ & 0.9800 \\
\hline $\mathrm{C} 26-\mathrm{H} 26$ & 0.9500 & $\mathrm{C} 64-\mathrm{H} 64 \mathrm{~A}$ & 0.9800 \\
\hline $\mathrm{C} 27-\mathrm{C} 28$ & $1.410(2)$ & C64-H64B & 0.9800 \\
\hline $\mathrm{C} 28-\mathrm{C} 29$ & $1.391(2)$ & $\mathrm{C} 64-\mathrm{H} 64 \mathrm{C}$ & 0.9800 \\
\hline $\mathrm{C} 29-\mathrm{C} 29 \mathrm{~A}$ & $1.387(2)$ & C65-H65A & 0.9800 \\
\hline $\mathrm{C} 29-\mathrm{H} 29$ & 0.9500 & $\mathrm{C} 65-\mathrm{H} 65 \mathrm{~B}$ & 0.9800 \\
\hline $\mathrm{C} 29 \mathrm{~A}-\mathrm{C} 30$ & $1.515(2)$ & $\mathrm{C} 65-\mathrm{H} 65 \mathrm{C}$ & 0.9800 \\
\hline $\mathrm{C} 30-\mathrm{C} 31$ & $1.541(2)$ & C66-H66A & 0.9800 \\
\hline $\mathrm{C} 30-\mathrm{C} 30 \mathrm{~A}$ & $1.566(2)$ & C66-H66B & 0.9800 \\
\hline $\mathrm{C} 30-\mathrm{H} 30$ & 1.0000 & C66-H66C & 0.9800 \\
\hline $\mathrm{C} 30 \mathrm{~A}-\mathrm{H} 30 \mathrm{~A}$ & 1.0000 & & \\
\hline $\mathrm{C} 17-\mathrm{O} 2-\mathrm{C} 18$ & $115.32(13)$ & $\mathrm{H} 31 \mathrm{~A}-\mathrm{C} 31-\mathrm{H} 31 \mathrm{~B}$ & 108.1 \\
\hline
\end{tabular}




\begin{tabular}{|c|c|}
\hline $\mathrm{C} 5-\mathrm{O} 3-\mathrm{C} 21$ & $116.44(13)$ \\
\hline $\mathrm{C} 6-\mathrm{O} 4-\mathrm{C} 20$ & $117.24(13)$ \\
\hline $\mathrm{C} 2-\mathrm{C} 1-\mathrm{C} 11$ & $131.73(15)$ \\
\hline $\mathrm{C} 2-\mathrm{C} 1-\mathrm{C} 8 \mathrm{~A}$ & $108.29(13)$ \\
\hline $\mathrm{C} 11-\mathrm{C} 1-\mathrm{C} 8 \mathrm{~A}$ & $119.97(13)$ \\
\hline $\mathrm{C} 1-\mathrm{C} 2-\mathrm{C} 17$ & $131.57(15)$ \\
\hline $\mathrm{C} 1-\mathrm{C} 2-\mathrm{C} 3$ & $109.17(13)$ \\
\hline $\mathrm{C} 17-\mathrm{C} 2-\mathrm{C} 3$ & $118.94(13)$ \\
\hline $\mathrm{N} 10-\mathrm{C} 3-\mathrm{C} 2$ & $111.07(13)$ \\
\hline $\mathrm{N} 10-\mathrm{C} 3-\mathrm{C} 3 \mathrm{~A}$ & $109.91(12)$ \\
\hline $\mathrm{C} 2-\mathrm{C} 3-\mathrm{C} 3 \mathrm{~A}$ & $101.02(12)$ \\
\hline $\mathrm{N} 10-\mathrm{C} 3-\mathrm{H} 3$ & 111.5 \\
\hline $\mathrm{C} 2-\mathrm{C} 3-\mathrm{H} 3$ & 111.5 \\
\hline $\mathrm{C} 3 \mathrm{~A}-\mathrm{C} 3-\mathrm{H} 3$ & 111.5 \\
\hline $\mathrm{C} 3 \mathrm{~B}-\mathrm{C} 3 \mathrm{~A}-\mathrm{C} 19$ & $114.39(13)$ \\
\hline $\mathrm{C} 3 \mathrm{~B}-\mathrm{C} 3 \mathrm{~A}-\mathrm{C} 8 \mathrm{~A}$ & $102.28(12)$ \\
\hline $\mathrm{C} 19-\mathrm{C} 3 \mathrm{~A}-\mathrm{C} 8 \mathrm{~A}$ & $115.55(13)$ \\
\hline $\mathrm{C} 3 \mathrm{~B}-\mathrm{C} 3 \mathrm{~A}-\mathrm{C} 3$ & $114.21(13)$ \\
\hline $\mathrm{C} 19-\mathrm{C} 3 \mathrm{~A}-\mathrm{C} 3$ & $111.78(13)$ \\
\hline $\mathrm{C} 8 \mathrm{~A}-\mathrm{C} 3 \mathrm{~A}-\mathrm{C} 3$ & $97.13(12)$ \\
\hline $\mathrm{C} 7 \mathrm{~A}-\mathrm{C} 3 \mathrm{~B}-\mathrm{C} 4$ & $121.05(14)$ \\
\hline $\mathrm{C} 7 \mathrm{~A}-\mathrm{C} 3 \mathrm{~B}-\mathrm{C} 3 \mathrm{~A}$ & $109.34(14)$ \\
\hline $\mathrm{C} 4-\mathrm{C} 3 \mathrm{~B}-\mathrm{C} 3 \mathrm{~A}$ & $129.61(15)$ \\
\hline $\mathrm{C} 3 \mathrm{~B}-\mathrm{C} 4-\mathrm{C} 5$ & $118.62(15)$ \\
\hline $\mathrm{C} 3 \mathrm{~B}-\mathrm{C} 4-\mathrm{H} 4$ & 120.7 \\
\hline $\mathrm{C} 5-\mathrm{C} 4-\mathrm{H} 4$ & 120.7 \\
\hline $\mathrm{O} 3-\mathrm{C} 5-\mathrm{C} 4$ & $124.74(15)$ \\
\hline $\mathrm{O} 3-\mathrm{C} 5-\mathrm{C} 6$ & $115.02(14)$ \\
\hline $\mathrm{C} 4-\mathrm{C} 5-\mathrm{C} 6$ & $120.24(15)$ \\
\hline $\mathrm{O} 4-\mathrm{C} 6-\mathrm{C} 7$ & $125.03(15)$ \\
\hline $\mathrm{O} 4-\mathrm{C} 6-\mathrm{C} 5$ & $114.44(14)$ \\
\hline $\mathrm{C} 7-\mathrm{C} 6-\mathrm{C} 5$ & $120.52(14)$ \\
\hline $\mathrm{C} 7 \mathrm{~A}-\mathrm{C} 7-\mathrm{C} 6$ & $118.45(15)$ \\
\hline $\mathrm{C} 7 \mathrm{~A}-\mathrm{C} 7-\mathrm{H} 7$ & 120.8 \\
\hline $\mathrm{C} 6-\mathrm{C} 7-\mathrm{H} 7$ & 120.8 \\
\hline $\mathrm{C} 3 \mathrm{~B}-\mathrm{C} 7 \mathrm{~A}-\mathrm{C} 7$ & $121.01(15)$ \\
\hline $\mathrm{C} 3 \mathrm{~B}-\mathrm{C} 7 \mathrm{~A}-\mathrm{C} 8$ & $109.04(13)$ \\
\hline $\mathrm{C} 7-\mathrm{C} 7 \mathrm{~A}-\mathrm{C} 8$ & $129.64(15)$ \\
\hline $\mathrm{C} 7 \mathrm{~A}-\mathrm{C} 8-\mathrm{C} 9$ & $107.86(13)$ \\
\hline $\mathrm{C} 7 \mathrm{~A}-\mathrm{C} 8-\mathrm{C} 8 \mathrm{~A}$ & $101.22(13)$ \\
\hline $\mathrm{C} 9-\mathrm{C} 8-\mathrm{C} 8 \mathrm{~A}$ & $108.69(12)$ \\
\hline $\mathrm{C} 7 \mathrm{~A}-\mathrm{C} 8-\mathrm{H} 8$ & 112.8 \\
\hline $\mathrm{C} 9-\mathrm{C} 8-\mathrm{H} 8$ & 112.8 \\
\hline $\mathrm{C} 8 \mathrm{~A}-\mathrm{C} 8-\mathrm{H} 8$ & 112.8 \\
\hline $\mathrm{C} 1-\mathrm{C} 8 \mathrm{~A}-\mathrm{C} 3 \mathrm{~A}$ & $102.94(12)$ \\
\hline $\mathrm{C} 1-\mathrm{C} 8 \mathrm{~A}-\mathrm{C} 8$ & $115.81(14)$ \\
\hline $\mathrm{C} 3 \mathrm{~A}-\mathrm{C} 8 \mathrm{~A}-\mathrm{C} 8$ & $100.12(12)$ \\
\hline $\mathrm{C} 1-\mathrm{C} 8 \mathrm{~A}-\mathrm{H} 8 \mathrm{~A}$ & 112.3 \\
\hline
\end{tabular}

\begin{tabular}{|c|c|}
\hline $\mathrm{C} 44-\mathrm{N} 32-\mathrm{C} 31$ & $111.03(13)$ \\
\hline $\mathrm{C} 44-\mathrm{N} 32-\mathrm{C} 25$ & $112.34(12)$ \\
\hline $\mathrm{C} 31-\mathrm{N} 32-\mathrm{C} 25$ & $112.55(12)$ \\
\hline $\mathrm{C} 34-\mathrm{C} 33-\mathrm{C} 38$ & $117.90(15)$ \\
\hline $\mathrm{C} 34-\mathrm{C} 33-\mathrm{C} 23$ & $123.82(15)$ \\
\hline $\mathrm{C} 38-\mathrm{C} 33-\mathrm{C} 23$ & $118.26(14)$ \\
\hline $\mathrm{C} 35-\mathrm{C} 34-\mathrm{C} 33$ & $120.70(16)$ \\
\hline $\mathrm{C} 35-\mathrm{C} 34-\mathrm{H} 34$ & 119.6 \\
\hline $\mathrm{C} 33-\mathrm{C} 34-\mathrm{H} 34$ & 119.6 \\
\hline $\mathrm{C} 36-\mathrm{C} 35-\mathrm{C} 34$ & $120.82(16)$ \\
\hline $\mathrm{C} 36-\mathrm{C} 35-\mathrm{H} 35$ & 119.6 \\
\hline $\mathrm{C} 34-\mathrm{C} 35-\mathrm{H} 35$ & 119.6 \\
\hline $\mathrm{C} 35-\mathrm{C} 36-\mathrm{C} 37$ & $119.05(16)$ \\
\hline $\mathrm{C} 35-\mathrm{C} 36-\mathrm{H} 36$ & 120.5 \\
\hline $\mathrm{C} 37-\mathrm{C} 36-\mathrm{H} 36$ & 120.5 \\
\hline $\mathrm{C} 38-\mathrm{C} 37-\mathrm{C} 36$ & $120.35(16)$ \\
\hline $\mathrm{C} 38-\mathrm{C} 37-\mathrm{H} 37$ & 119.8 \\
\hline $\mathrm{C} 36-\mathrm{C} 37-\mathrm{H} 37$ & 119.8 \\
\hline $\mathrm{C} 37-\mathrm{C} 38-\mathrm{C} 33$ & $121.11(15)$ \\
\hline $\mathrm{C} 37-\mathrm{C} 38-\mathrm{H} 38$ & 119.4 \\
\hline $\mathrm{C} 33-\mathrm{C} 38-\mathrm{H} 38$ & 119.4 \\
\hline $\mathrm{O} 5-\mathrm{C} 39-\mathrm{O} 6$ & $122.77(15)$ \\
\hline $\mathrm{O} 5-\mathrm{C} 39-\mathrm{C} 24$ & $123.36(15)$ \\
\hline $\mathrm{O} 6-\mathrm{C} 39-\mathrm{C} 24$ & $113.86(13)$ \\
\hline $\mathrm{O} 6-\mathrm{C} 40-\mathrm{H} 40 \mathrm{~A}$ & 109.5 \\
\hline $\mathrm{O} 6-\mathrm{C} 40-\mathrm{H} 40 \mathrm{~B}$ & 109.5 \\
\hline $\mathrm{H} 40 \mathrm{~A}-\mathrm{C} 40-\mathrm{H} 40 \mathrm{~B}$ & 109.5 \\
\hline $\mathrm{O} 6-\mathrm{C} 40-\mathrm{H} 40 \mathrm{C}$ & 109.5 \\
\hline $\mathrm{H} 40 \mathrm{~A}-\mathrm{C} 40-\mathrm{H} 40 \mathrm{C}$ & 109.5 \\
\hline $\mathrm{H} 40 \mathrm{~B}-\mathrm{C} 40-\mathrm{H} 40 \mathrm{C}$ & 109.5 \\
\hline $\mathrm{C} 25 \mathrm{~A}-\mathrm{C} 41-\mathrm{H} 41 \mathrm{~A}$ & 109.5 \\
\hline $\mathrm{C} 25 \mathrm{~A}-\mathrm{C} 41-\mathrm{H} 41 \mathrm{~B}$ & 109.5 \\
\hline $\mathrm{H} 41 \mathrm{~A}-\mathrm{C} 41-\mathrm{H} 41 \mathrm{~B}$ & 109.5 \\
\hline $\mathrm{C} 25 \mathrm{~A}-\mathrm{C} 41-\mathrm{H} 41 \mathrm{C}$ & 109.5 \\
\hline $\mathrm{H} 41 \mathrm{~A}-\mathrm{C} 41-\mathrm{H} 41 \mathrm{C}$ & 109.5 \\
\hline $\mathrm{H} 41 \mathrm{~B}-\mathrm{C} 41-\mathrm{H} 41 \mathrm{C}$ & 109.5 \\
\hline $\mathrm{O} 8-\mathrm{C} 42-\mathrm{H} 42 \mathrm{~A}$ & 109.5 \\
\hline $\mathrm{O} 8-\mathrm{C} 42-\mathrm{H} 42 \mathrm{~B}$ & 109.5 \\
\hline $\mathrm{H} 42 \mathrm{~A}-\mathrm{C} 42-\mathrm{H} 42 \mathrm{~B}$ & 109.5 \\
\hline $\mathrm{O} 8-\mathrm{C} 42-\mathrm{H} 42 \mathrm{C}$ & 109.5 \\
\hline $\mathrm{H} 42 \mathrm{~A}-\mathrm{C} 42-\mathrm{H} 42 \mathrm{C}$ & 109.5 \\
\hline $\mathrm{H} 42 \mathrm{~B}-\mathrm{C} 42-\mathrm{H} 42 \mathrm{C}$ & 109.5 \\
\hline $\mathrm{O} 7-\mathrm{C} 43-\mathrm{H} 43 \mathrm{~A}$ & 109.5 \\
\hline $\mathrm{O} 7-\mathrm{C} 43-\mathrm{H} 43 \mathrm{~B}$ & 109.5 \\
\hline $\mathrm{H} 43 \mathrm{~A}-\mathrm{C} 43-\mathrm{H} 43 \mathrm{~B}$ & 109.5 \\
\hline $\mathrm{O} 7-\mathrm{C} 43-\mathrm{H} 43 \mathrm{C}$ & 109.5 \\
\hline $\mathrm{H} 43 \mathrm{~A}-\mathrm{C} 43-\mathrm{H} 43 \mathrm{C}$ & 109.5 \\
\hline $\mathrm{H} 43 \mathrm{~B}-\mathrm{C} 43-\mathrm{H} 43 \mathrm{C}$ & 109.5 \\
\hline
\end{tabular}




$\begin{array}{ll}\text { C3A-C8A-H8A } & 112.3 \\ \text { C8-C8A-H8A } & 112.3 \\ \text { N10-C9-C } & 110.22(13) \\ \text { N10-C9-H9A } & 109.6 \\ \text { C8-C9-H9A } & 109.6 \\ \text { N10-C9-H9B } & 109.6 \\ \text { C8-C9-H9B } & 109.6 \\ \text { H9A-C9-H9B } & 108.1 \\ \text { C22-N10-C9 } & 110.70(13) \\ \text { C22-N10-C3 } & 113.17(12) \\ \text { C9-N10-C3 } & 112.43(12) \\ \text { C12-C11-C16 } & 118.24(15) \\ \text { C12-C11-C1 } & 122.36(15) \\ \text { C16-C11-C1 } & 119.36(14) \\ \text { C13-C12-C11 } & 121.27(17) \\ \text { C13-C12-H12 } & 119.4 \\ \text { C11-C12-H12 } & 119.4 \\ \text { C12-C13-C14 } & 120.25(16) \\ \text { C12-C13-H13 } & 119.9 \\ \text { C14-C13-H13 } & 119.9 \\ \text { C15-C14-C13 } & 119.00(16) \\ \text { C15-C14-H14 } & 120.5 \\ \text { C13-C14-H14 } & 120.5 \\ \text { C14-C15-C16 } & 120.99(17) \\ \text { C14-C15-H15 } & 119.5 \\ \text { C16-C15-H15 } & 119.5 \\ \text { C15-C16-C11 } & 120.19(15) \\ \text { C15-C16-H16 } & 119.9 \\ \text { C11-C16-H16 } & 119.9 \\ \text { O1-C17-O2 } & 122.95(15) \\ \text { O1-C17-C2 } & 122.93(14) \\ \text { O2-C17-C2 } & 114.11(14) \\ \text { O2-C18-H18A } & 109.5 \\ \text { O2-C18-H18B } & 109.5 \\ \text { H18A-C18-H18B } & 109.5 \\ \text { O2-C18-H18C } & 109.5 \\ \text { H18A-C18-H18C } & 109.5 \\ \text { H18B-C18-H18C } & 109.5 \\ \text { C3A-C19-H19A } & 109.5 \\ \text { C3A-C19-H19B } & 109.5 \\ \text { H19A-C19-H19B } & 109.5 \\ \text { C3A-C19-H19C } & 109.5 \\ \text { H19A-C19-H19C } & 109.5 \\ \text { H19B-C19-H19C } & 109.5 \\ \text { O4-C20-C20-H20A } & \\ \text { O4-C20-H20B } & \\ & \end{array}$

\begin{tabular}{|c|c|}
\hline $\mathrm{N} 32-\mathrm{C} 44-\mathrm{H} 44 \mathrm{~A}$ & 109.5 \\
\hline $\mathrm{N} 32-\mathrm{C} 44-\mathrm{H} 44 \mathrm{~B}$ & 109.5 \\
\hline $\mathrm{H} 44 \mathrm{~A}-\mathrm{C} 44-\mathrm{H} 44 \mathrm{~B}$ & 109.5 \\
\hline $\mathrm{N} 32-\mathrm{C} 44-\mathrm{H} 44 \mathrm{C}$ & 109.5 \\
\hline $\mathrm{H} 44 \mathrm{~A}-\mathrm{C} 44-\mathrm{H} 44 \mathrm{C}$ & 109.5 \\
\hline $\mathrm{H} 44 \mathrm{~B}-\mathrm{C} 44-\mathrm{H} 44 \mathrm{C}$ & 109.5 \\
\hline $\mathrm{C} 61-\mathrm{O} 10-\mathrm{C} 62$ & $115.94(13)$ \\
\hline $\mathrm{C} 49-\mathrm{O} 11-\mathrm{C} 65$ & $117.15(14)$ \\
\hline $\mathrm{C} 50-\mathrm{O} 12-\mathrm{C} 64$ & $117.71(15)$ \\
\hline $\mathrm{C} 46-\mathrm{C} 45-\mathrm{C} 55$ & $132.22(15)$ \\
\hline $\mathrm{C} 46-\mathrm{C} 45-\mathrm{C} 52 \mathrm{~A}$ & $108.11(13)$ \\
\hline $\mathrm{C} 55-\mathrm{C} 45-\mathrm{C} 52 \mathrm{~A}$ & $119.67(14)$ \\
\hline $\mathrm{C} 45-\mathrm{C} 46-\mathrm{C} 61$ & $131.83(14)$ \\
\hline $\mathrm{C} 45-\mathrm{C} 46-\mathrm{C} 47$ & $108.93(13)$ \\
\hline $\mathrm{C} 61-\mathrm{C} 46-\mathrm{C} 47$ & $118.88(13)$ \\
\hline N54-C47-C46 & $110.80(13)$ \\
\hline $\mathrm{N} 54-\mathrm{C} 47-\mathrm{C} 47 \mathrm{~A}$ & $110.20(12)$ \\
\hline $\mathrm{C} 46-\mathrm{C} 47-\mathrm{C} 47 \mathrm{~A}$ & $101.34(12)$ \\
\hline N54-C47-H47 & 111.4 \\
\hline $\mathrm{C} 46-\mathrm{C} 47-\mathrm{H} 47$ & 111.4 \\
\hline $\mathrm{C} 47 \mathrm{~A}-\mathrm{C} 47-\mathrm{H} 47$ & 111.4 \\
\hline $\mathrm{C} 47 \mathrm{~B}-\mathrm{C} 47 \mathrm{~A}-\mathrm{C} 63$ & $114.94(14)$ \\
\hline $\mathrm{C} 47 \mathrm{~B}-\mathrm{C} 47 \mathrm{~A}-\mathrm{C} 52 \mathrm{~A}$ & $102.44(13)$ \\
\hline $\mathrm{C} 63-\mathrm{C} 47 \mathrm{~A}-\mathrm{C} 52 \mathrm{~A}$ & $116.19(14)$ \\
\hline $\mathrm{C} 47 \mathrm{~B}-\mathrm{C} 47 \mathrm{~A}-\mathrm{C} 47$ & $114.04(13)$ \\
\hline $\mathrm{C} 63-\mathrm{C} 47 \mathrm{~A}-\mathrm{C} 47$ & $111.00(13)$ \\
\hline $\mathrm{C} 52 \mathrm{~A}-\mathrm{C} 47 \mathrm{~A}-\mathrm{C} 47$ & $96.70(13)$ \\
\hline $\mathrm{C} 51 \mathrm{~A}-\mathrm{C} 47 \mathrm{~B}-\mathrm{C} 48$ & $121.09(15)$ \\
\hline $\mathrm{C} 51 \mathrm{~A}-\mathrm{C} 47 \mathrm{~B}-\mathrm{C} 47 \mathrm{~A}$ & $109.35(14)$ \\
\hline $\mathrm{C} 48-\mathrm{C} 47 \mathrm{~B}-\mathrm{C} 47 \mathrm{~A}$ & $129.54(15)$ \\
\hline $\mathrm{C} 49-\mathrm{C} 48-\mathrm{C} 47 \mathrm{~B}$ & $118.67(16)$ \\
\hline $\mathrm{C} 49-\mathrm{C} 48-\mathrm{H} 48$ & 120.7 \\
\hline $\mathrm{C} 47 \mathrm{~B}-\mathrm{C} 48-\mathrm{H} 48$ & 120.7 \\
\hline $\mathrm{O} 11-\mathrm{C} 49-\mathrm{C} 48$ & $124.86(16)$ \\
\hline $\mathrm{O} 11-\mathrm{C} 49-\mathrm{C} 50$ & $115.17(14)$ \\
\hline $\mathrm{C} 48-\mathrm{C} 49-\mathrm{C} 50$ & $119.96(16)$ \\
\hline $\mathrm{O} 12-\mathrm{C} 50-\mathrm{C} 51$ & $124.83(17)$ \\
\hline $\mathrm{O} 12-\mathrm{C} 50-\mathrm{C} 49$ & $114.11(15)$ \\
\hline $\mathrm{C} 51-\mathrm{C} 50-\mathrm{C} 49$ & $121.06(15)$ \\
\hline $\mathrm{C} 51 \mathrm{~A}-\mathrm{C} 51-\mathrm{C} 50$ & $118.25(17)$ \\
\hline $\mathrm{C} 51 \mathrm{~A}-\mathrm{C} 51-\mathrm{H} 51$ & 120.9 \\
\hline $\mathrm{C} 50-\mathrm{C} 51-\mathrm{H} 51$ & 120.9 \\
\hline $\mathrm{C} 47 \mathrm{~B}-\mathrm{C} 51 \mathrm{~A}-\mathrm{C} 51$ & $120.81(16)$ \\
\hline $\mathrm{C} 47 \mathrm{~B}-\mathrm{C} 51 \mathrm{~A}-\mathrm{C} 52$ & $109.30(14)$ \\
\hline $\mathrm{C} 51-\mathrm{C} 51 \mathrm{~A}-\mathrm{C} 52$ & $129.37(16)$ \\
\hline $\mathrm{C} 51 \mathrm{~A}-\mathrm{C} 52-\mathrm{C} 53$ & $107.87(13)$ \\
\hline $\mathrm{C} 51 \mathrm{~A}-\mathrm{C} 52-\mathrm{C} 52 \mathrm{~A}$ & $101.29(13)$ \\
\hline $\mathrm{C} 53-\mathrm{C} 52-\mathrm{C} 52 \mathrm{~A}$ & $108.58(13)$ \\
\hline
\end{tabular}




\begin{tabular}{|c|c|c|c|}
\hline $\mathrm{H} 20 \mathrm{~A}-\mathrm{C} 20-\mathrm{H} 20 \mathrm{C}$ & 109.5 & $\mathrm{C} 51 \mathrm{~A}-\mathrm{C} 52-\mathrm{H} 52$ & 112.8 \\
\hline $\mathrm{H} 20 \mathrm{~B}-\mathrm{C} 20-\mathrm{H} 20 \mathrm{C}$ & 109.5 & $\mathrm{C} 53-\mathrm{C} 52-\mathrm{H} 52$ & 112.8 \\
\hline $\mathrm{O} 3-\mathrm{C} 21-\mathrm{H} 21 \mathrm{~A}$ & 109.5 & $\mathrm{C} 52 \mathrm{~A}-\mathrm{C} 52-\mathrm{H} 52$ & 112.8 \\
\hline $\mathrm{O} 3-\mathrm{C} 21-\mathrm{H} 21 \mathrm{~B}$ & 109.5 & $\mathrm{C} 45-\mathrm{C} 52 \mathrm{~A}-\mathrm{C} 47 \mathrm{~A}$ & $103.53(12)$ \\
\hline $\mathrm{H} 21 \mathrm{~A}-\mathrm{C} 21-\mathrm{H} 21 \mathrm{~B}$ & 109.5 & $\mathrm{C} 45-\mathrm{C} 52 \mathrm{~A}-\mathrm{C} 52$ & $115.48(14)$ \\
\hline $\mathrm{O} 3-\mathrm{C} 21-\mathrm{H} 21 \mathrm{C}$ & 109.5 & $\mathrm{C} 47 \mathrm{~A}-\mathrm{C} 52 \mathrm{~A}-\mathrm{C} 52$ & $99.98(12)$ \\
\hline $\mathrm{H} 21 \mathrm{~A}-\mathrm{C} 21-\mathrm{H} 21 \mathrm{C}$ & 109.5 & $\mathrm{C} 45-\mathrm{C} 52 \mathrm{~A}-\mathrm{H} 52 \mathrm{~A}$ & 112.3 \\
\hline $\mathrm{H} 21 \mathrm{~B}-\mathrm{C} 21-\mathrm{H} 21 \mathrm{C}$ & 109.5 & $\mathrm{C} 47 \mathrm{~A}-\mathrm{C} 52 \mathrm{~A}-\mathrm{H} 52 \mathrm{~A}$ & 112.3 \\
\hline $\mathrm{N} 10-\mathrm{C} 22-\mathrm{H} 22 \mathrm{~A}$ & 109.5 & $\mathrm{C} 52-\mathrm{C} 52 \mathrm{~A}-\mathrm{H} 52 \mathrm{~A}$ & 112.3 \\
\hline $\mathrm{N} 10-\mathrm{C} 22-\mathrm{H} 22 \mathrm{~B}$ & 109.5 & $\mathrm{~N} 54-\mathrm{C} 53-\mathrm{C} 52$ & $110.03(14)$ \\
\hline $\mathrm{H} 22 \mathrm{~A}-\mathrm{C} 22-\mathrm{H} 22 \mathrm{~B}$ & 109.5 & N54-C53-H53A & 109.7 \\
\hline $\mathrm{N} 10-\mathrm{C} 22-\mathrm{H} 22 \mathrm{C}$ & 109.5 & C52-C53-H53A & 109.7 \\
\hline $\mathrm{H} 22 \mathrm{~A}-\mathrm{C} 22-\mathrm{H} 22 \mathrm{C}$ & 109.5 & N54-C53-H53B & 109.7 \\
\hline $\mathrm{H} 22 \mathrm{~B}-\mathrm{C} 22-\mathrm{H} 22 \mathrm{C}$ & 109.5 & С $52-\mathrm{C} 53-\mathrm{H} 53 \mathrm{~B}$ & 109.7 \\
\hline $\mathrm{C} 39-\mathrm{O} 6-\mathrm{C} 40$ & $116.17(13)$ & $\mathrm{H} 53 \mathrm{~A}-\mathrm{C} 53-\mathrm{H} 53 \mathrm{~B}$ & 108.2 \\
\hline $\mathrm{C} 27-\mathrm{O} 7-\mathrm{C} 43$ & $116.59(13)$ & $\mathrm{C} 66-\mathrm{N} 54-\mathrm{C} 53$ & $111.07(13)$ \\
\hline $\mathrm{C} 28-\mathrm{O} 8-\mathrm{C} 42$ & $115.06(12)$ & $\mathrm{C} 66-\mathrm{N} 54-\mathrm{C} 47$ & $112.94(13)$ \\
\hline $\mathrm{C} 24-\mathrm{C} 23-\mathrm{C} 33$ & $132.29(14)$ & $\mathrm{C} 53-\mathrm{N} 54-\mathrm{C} 47$ & $112.27(12)$ \\
\hline $\mathrm{C} 24-\mathrm{C} 23-\mathrm{C} 30 \mathrm{~A}$ & $107.67(13)$ & $\mathrm{C} 56-\mathrm{C} 55-\mathrm{C} 60$ & $118.20(15)$ \\
\hline $\mathrm{C} 33-\mathrm{C} 23-\mathrm{C} 30 \mathrm{~A}$ & $119.97(13)$ & $\mathrm{C} 56-\mathrm{C} 55-\mathrm{C} 45$ & $123.61(15)$ \\
\hline $\mathrm{C} 23-\mathrm{C} 24-\mathrm{C} 39$ & $131.03(15)$ & $\mathrm{C} 60-\mathrm{C} 55-\mathrm{C} 45$ & $118.14(14)$ \\
\hline $\mathrm{C} 23-\mathrm{C} 24-\mathrm{C} 25$ & $109.29(13)$ & $\mathrm{C} 57-\mathrm{C} 56-\mathrm{C} 55$ & $120.68(16)$ \\
\hline $\mathrm{C} 39-\mathrm{C} 24-\mathrm{C} 25$ & $119.26(13)$ & $\mathrm{C} 57-\mathrm{C} 56-\mathrm{H} 56$ & 119.7 \\
\hline $\mathrm{N} 32-\mathrm{C} 25-\mathrm{C} 24$ & $110.41(13)$ & $\mathrm{C} 55-\mathrm{C} 56-\mathrm{H} 56$ & 119.7 \\
\hline $\mathrm{N} 32-\mathrm{C} 25-\mathrm{C} 25 \mathrm{~A}$ & $109.79(12)$ & $\mathrm{C} 58-\mathrm{C} 57-\mathrm{C} 56$ & $120.66(15)$ \\
\hline $\mathrm{C} 24-\mathrm{C} 25-\mathrm{C} 25 \mathrm{~A}$ & $101.45(12)$ & $\mathrm{C} 58-\mathrm{C} 57-\mathrm{H} 57$ & 119.7 \\
\hline $\mathrm{N} 32-\mathrm{C} 25-\mathrm{H} 25$ & 111.6 & $\mathrm{C} 56-\mathrm{C} 57-\mathrm{H} 57$ & 119.7 \\
\hline $\mathrm{C} 24-\mathrm{C} 25-\mathrm{H} 25$ & 111.6 & $\mathrm{C} 57-\mathrm{C} 58-\mathrm{C} 59$ & $119.18(15)$ \\
\hline $\mathrm{C} 25 \mathrm{~A}-\mathrm{C} 25-\mathrm{H} 25$ & 111.6 & $\mathrm{C} 57-\mathrm{C} 58-\mathrm{H} 58$ & 120.4 \\
\hline $\mathrm{C} 25 \mathrm{~B}-\mathrm{C} 25 \mathrm{~A}-\mathrm{C} 41$ & $114.38(13)$ & $\mathrm{C} 59-\mathrm{C} 58-\mathrm{H} 58$ & 120.4 \\
\hline $\mathrm{C} 25 \mathrm{~B}-\mathrm{C} 25 \mathrm{~A}-\mathrm{C} 30 \mathrm{~A}$ & $102.27(12)$ & $\mathrm{C} 60-\mathrm{C} 59-\mathrm{C} 58$ & $120.32(16)$ \\
\hline $\mathrm{C} 41-\mathrm{C} 25 \mathrm{~A}-\mathrm{C} 30 \mathrm{~A}$ & $116.53(13)$ & $\mathrm{C} 60-\mathrm{C} 59-\mathrm{H} 59$ & 119.8 \\
\hline $\mathrm{C} 25 \mathrm{~B}-\mathrm{C} 25 \mathrm{~A}-\mathrm{C} 25$ & $114.79(13)$ & С58-C59-H59 & 119.8 \\
\hline $\mathrm{C} 41-\mathrm{C} 25 \mathrm{~A}-\mathrm{C} 25$ & $110.51(12)$ & $\mathrm{C} 59-\mathrm{C} 60-\mathrm{C} 55$ & $120.90(15)$ \\
\hline $\mathrm{C} 30 \mathrm{~A}-\mathrm{C} 25 \mathrm{~A}-\mathrm{C} 25$ & $97.07(12)$ & $\mathrm{C} 59-\mathrm{C} 60-\mathrm{H} 60$ & 119.5 \\
\hline $\mathrm{C} 26-\mathrm{C} 25 \mathrm{~B}-\mathrm{C} 29 \mathrm{~A}$ & $120.83(14)$ & $\mathrm{C} 55-\mathrm{C} 60-\mathrm{H} 60$ & 119.5 \\
\hline $\mathrm{C} 26-\mathrm{C} 25 \mathrm{~B}-\mathrm{C} 25 \mathrm{~A}$ & $130.04(14)$ & $\mathrm{O} 9-\mathrm{C} 61-\mathrm{O} 10$ & $123.04(15)$ \\
\hline $\mathrm{C} 29 \mathrm{~A}-\mathrm{C} 25 \mathrm{~B}-\mathrm{C} 25 \mathrm{~A}$ & $109.12(13)$ & $\mathrm{O} 9-\mathrm{C} 61-\mathrm{C} 46$ & $122.97(15)$ \\
\hline $\mathrm{C} 25 \mathrm{~B}-\mathrm{C} 26-\mathrm{C} 27$ & $118.64(15)$ & $\mathrm{O} 10-\mathrm{C} 61-\mathrm{C} 46$ & $113.99(14)$ \\
\hline $\mathrm{C} 25 \mathrm{~B}-\mathrm{C} 26-\mathrm{H} 26$ & 120.7 & $\mathrm{O} 10-\mathrm{C} 62-\mathrm{H} 62 \mathrm{~A}$ & 109.5 \\
\hline $\mathrm{C} 27-\mathrm{C} 26-\mathrm{H} 26$ & 120.7 & $\mathrm{O} 10-\mathrm{C} 62-\mathrm{H} 62 \mathrm{~B}$ & 109.5 \\
\hline $\mathrm{O} 7-\mathrm{C} 27-\mathrm{C} 26$ & $124.50(15)$ & $\mathrm{H} 62 \mathrm{~A}-\mathrm{C} 62-\mathrm{H} 62 \mathrm{~B}$ & 109.5 \\
\hline $\mathrm{O} 7-\mathrm{C} 27-\mathrm{C} 28$ & $115.37(14)$ & $\mathrm{O} 10-\mathrm{C} 62-\mathrm{H} 62 \mathrm{C}$ & 109.5 \\
\hline $\mathrm{C} 26-\mathrm{C} 27-\mathrm{C} 28$ & $120.13(14)$ & $\mathrm{H} 62 \mathrm{~A}-\mathrm{C} 62-\mathrm{H} 62 \mathrm{C}$ & 109.5 \\
\hline $\mathrm{C} 29-\mathrm{C} 28-\mathrm{O} 8$ & $118.79(14)$ & $\mathrm{H} 62 \mathrm{~B}-\mathrm{C} 62-\mathrm{H} 62 \mathrm{C}$ & 109.5 \\
\hline $\mathrm{C} 29-\mathrm{C} 28-\mathrm{C} 27$ & $120.49(14)$ & $\mathrm{C} 47 \mathrm{~A}-\mathrm{C} 63-\mathrm{H} 63 \mathrm{~A}$ & 109.5 \\
\hline $\mathrm{O} 8-\mathrm{C} 28-\mathrm{C} 27$ & $120.50(14)$ & $\mathrm{C} 47 \mathrm{~A}-\mathrm{C} 63-\mathrm{H} 63 \mathrm{~B}$ & 109.5 \\
\hline $\mathrm{C} 29 \mathrm{~A}-\mathrm{C} 29-\mathrm{C} 28$ & $118.94(15)$ & $\mathrm{H} 63 \mathrm{~A}-\mathrm{C} 63-\mathrm{H} 63 \mathrm{~B}$ & 109.5 \\
\hline
\end{tabular}




\begin{tabular}{|c|c|}
\hline $\mathrm{C} 29 \mathrm{~A}-\mathrm{C} 29-\mathrm{H} 29$ & 120.5 \\
\hline $\mathrm{C} 28-\mathrm{C} 29-\mathrm{H} 29$ & 120.5 \\
\hline $\mathrm{C} 29-\mathrm{C} 29 \mathrm{~A}-\mathrm{C} 25 \mathrm{~B}$ & $120.93(15)$ \\
\hline $\mathrm{C} 29-\mathrm{C} 29 \mathrm{~A}-\mathrm{C} 30$ & $129.89(15)$ \\
\hline $\mathrm{C} 25 \mathrm{~B}-\mathrm{C} 29 \mathrm{~A}-\mathrm{C} 30$ & $109.08(13)$ \\
\hline $\mathrm{C} 29 \mathrm{~A}-\mathrm{C} 30-\mathrm{C} 31$ & $108.47(13)$ \\
\hline $\mathrm{C} 29 \mathrm{~A}-\mathrm{C} 30-\mathrm{C} 30 \mathrm{~A}$ & $101.31(13)$ \\
\hline $\mathrm{C} 31-\mathrm{C} 30-\mathrm{C} 30 \mathrm{~A}$ & $108.45(12)$ \\
\hline $\mathrm{C} 29 \mathrm{~A}-\mathrm{C} 30-\mathrm{H} 30$ & 112.6 \\
\hline $\mathrm{C} 31-\mathrm{C} 30-\mathrm{H} 30$ & 112.6 \\
\hline $\mathrm{C} 30 \mathrm{~A}-\mathrm{C} 30-\mathrm{H} 30$ & 112.6 \\
\hline $\mathrm{C} 23-\mathrm{C} 30 \mathrm{~A}-\mathrm{C} 25 \mathrm{~A}$ & $104.16(12)$ \\
\hline $\mathrm{C} 23-\mathrm{C} 30 \mathrm{~A}-\mathrm{C} 30$ & $114.93(13)$ \\
\hline $\mathrm{C} 25 \mathrm{~A}-\mathrm{C} 30 \mathrm{~A}-\mathrm{C} 30$ & $99.80(12)$ \\
\hline $\mathrm{C} 23-\mathrm{C} 30 \mathrm{~A}-\mathrm{H} 30 \mathrm{~A}$ & 112.3 \\
\hline $\mathrm{C} 25 \mathrm{~A}-\mathrm{C} 30 \mathrm{~A}-\mathrm{H} 30 \mathrm{~A}$ & 112.3 \\
\hline $\mathrm{C} 30-\mathrm{C} 30 \mathrm{~A}-\mathrm{H} 30 \mathrm{~A}$ & 112.3 \\
\hline $\mathrm{N} 32-\mathrm{C} 31-\mathrm{C} 30$ & $110.63(13)$ \\
\hline $\mathrm{N} 32-\mathrm{C} 31-\mathrm{H} 31 \mathrm{~A}$ & 109.5 \\
\hline $\mathrm{C} 30-\mathrm{C} 31-\mathrm{H} 31 \mathrm{~A}$ & 109.5 \\
\hline $\mathrm{N} 32-\mathrm{C} 31-\mathrm{H} 31 \mathrm{~B}$ & 109.5 \\
\hline $\mathrm{C} 30-\mathrm{C} 31-\mathrm{H} 31 \mathrm{~B}$ & 109.5 \\
\hline $\mathrm{C} 11-\mathrm{C} 1-\mathrm{C} 2-\mathrm{C} 17$ & $-4.3(3)$ \\
\hline $\mathrm{C} 8 \mathrm{~A}-\mathrm{C} 1-\mathrm{C} 2-\mathrm{C} 17$ & $177.21(17)$ \\
\hline $\mathrm{C} 11-\mathrm{C} 1-\mathrm{C} 2-\mathrm{C} 3$ & $-177.48(16)$ \\
\hline $\mathrm{C} 8 \mathrm{~A}-\mathrm{C} 1-\mathrm{C} 2-\mathrm{C} 3$ & $3.99(18)$ \\
\hline $\mathrm{C} 1-\mathrm{C} 2-\mathrm{C} 3-\mathrm{N} 10$ & $85.35(16)$ \\
\hline $\mathrm{C} 17-\mathrm{C} 2-\mathrm{C} 3-\mathrm{N} 10$ & $-88.86(17)$ \\
\hline $\mathrm{C} 1-\mathrm{C} 2-\mathrm{C} 3-\mathrm{C} 3 \mathrm{~A}$ & $-31.19(17)$ \\
\hline $\mathrm{C} 17-\mathrm{C} 2-\mathrm{C} 3-\mathrm{C} 3 \mathrm{~A}$ & $154.60(14)$ \\
\hline $\mathrm{N} 10-\mathrm{C} 3-\mathrm{C} 3 \mathrm{~A}-\mathrm{C} 3 \mathrm{~B}$ & $32.76(18)$ \\
\hline $\mathrm{C} 2-\mathrm{C} 3-\mathrm{C} 3 \mathrm{~A}-\mathrm{C} 3 \mathrm{~B}$ & $150.15(13)$ \\
\hline $\mathrm{N} 10-\mathrm{C} 3-\mathrm{C} 3 \mathrm{~A}-\mathrm{C} 19$ & $164.63(13)$ \\
\hline $\mathrm{C} 2-\mathrm{C} 3-\mathrm{C} 3 \mathrm{~A}-\mathrm{C} 19$ & $-77.98(15)$ \\
\hline $\mathrm{N} 10-\mathrm{C} 3-\mathrm{C} 3 \mathrm{~A}-\mathrm{C} 8 \mathrm{~A}$ & $-74.17(14)$ \\
\hline $\mathrm{C} 2-\mathrm{C} 3-\mathrm{C} 3 \mathrm{~A}-\mathrm{C} 8 \mathrm{~A}$ & $43.22(14)$ \\
\hline $\mathrm{C} 19-\mathrm{C} 3 \mathrm{~A}-\mathrm{C} 3 \mathrm{~B}-\mathrm{C} 7 \mathrm{~A}$ & $149.06(14)$ \\
\hline $\mathrm{C} 8 \mathrm{~A}-\mathrm{C} 3 \mathrm{~A}-\mathrm{C} 3 \mathrm{~B}-\mathrm{C} 7 \mathrm{~A}$ & $23.36(17)$ \\
\hline $\mathrm{C} 3-\mathrm{C} 3 \mathrm{~A}-\mathrm{C} 3 \mathrm{~B}-\mathrm{C} 7 \mathrm{~A}$ & $-80.35(16)$ \\
\hline $\mathrm{C} 19-\mathrm{C} 3 \mathrm{~A}-\mathrm{C} 3 \mathrm{~B}-\mathrm{C} 4$ & $-30.9(2)$ \\
\hline $\mathrm{C} 8 \mathrm{~A}-\mathrm{C} 3 \mathrm{~A}-\mathrm{C} 3 \mathrm{~B}-\mathrm{C} 4$ & $-156.63(16)$ \\
\hline $\mathrm{C} 3-\mathrm{C} 3 \mathrm{~A}-\mathrm{C} 3 \mathrm{~B}-\mathrm{C} 4$ & $99.7(2)$ \\
\hline $\mathrm{C} 7 \mathrm{~A}-\mathrm{C} 3 \mathrm{~B}-\mathrm{C} 4-\mathrm{C} 5$ & $0.4(2)$ \\
\hline $\mathrm{C} 3 \mathrm{~A}-\mathrm{C} 3 \mathrm{~B}-\mathrm{C} 4-\mathrm{C} 5$ & $-179.63(15)$ \\
\hline $\mathrm{C} 21-\mathrm{O} 3-\mathrm{C} 5-\mathrm{C} 4$ & $10.8(2)$ \\
\hline $\mathrm{C} 21-\mathrm{O} 3-\mathrm{C} 5-\mathrm{C} 6$ & $-169.50(14)$ \\
\hline $\mathrm{C} 3 \mathrm{~B}-\mathrm{C} 4-\mathrm{C} 5-\mathrm{O} 3$ & $-177.74(15)$ \\
\hline
\end{tabular}

$\begin{array}{ll}\text { C47A-C63-H63C } & 109.5 \\ \text { H63A-C63-H63C } & 109.5 \\ \text { H63B-C63-H63C } & 109.5 \\ \text { O12-C64-H64A } & 109.5 \\ \text { O12-C64-H64B } & 109.5 \\ \text { H64A-C64-H64B } & 109.5 \\ \text { O12-C64-H64C } & 109.5 \\ \text { H64A-C64-H64C } & 109.5 \\ \text { H64B-C64-H64C } & 109.5 \\ \text { O11-C65-H65A } & 109.5 \\ \text { O11-C65-H65B } & 109.5 \\ \text { H65A-C65-H65B } & 109.5 \\ \text { O11-C65-H65C } & 109.5 \\ \text { H65A-C65-H65C } & 109.5 \\ \text { H65B-C65-H65C } & 109.5 \\ \text { N54-C66-H66A } & 109.5 \\ \text { N54-C66-H66B } & 109.5 \\ \text { H66A-C66-H66B } & 109.5 \\ \text { N54-C66-H66C } & 109.5 \\ \text { H66A-C66-H66C } & 109.5 \\ \text { H66B-C66-H66C } & 109.5\end{array}$

$\begin{array}{ll}\mathrm{C} 29-\mathrm{C} 29 \mathrm{~A}-\mathrm{C} 30-\mathrm{C} 30 \mathrm{~A} & 154.58(17) \\ \mathrm{C} 25 \mathrm{~B}-\mathrm{C} 29 \mathrm{~A}-\mathrm{C} 30-\mathrm{C} 30 \mathrm{~A} & -29.07(16) \\ \mathrm{C} 24-\mathrm{C} 23-\mathrm{C} 30 \mathrm{~A}-\mathrm{C} 25 \mathrm{~A} & 23.32(17) \\ \mathrm{C} 33-\mathrm{C} 23-\mathrm{C} 30 \mathrm{~A}-\mathrm{C} 25 \mathrm{~A} & -159.21(14) \\ \mathrm{C} 24-\mathrm{C} 23-\mathrm{C} 30 \mathrm{~A}-\mathrm{C} 30 & -84.78(16) \\ \mathrm{C} 33-\mathrm{C} 23-\mathrm{C} 30 \mathrm{~A}-\mathrm{C} 30 & 92.68(17) \\ \mathrm{C} 25 \mathrm{~B}-\mathrm{C} 25 \mathrm{~A}-\mathrm{C} 30 \mathrm{~A}-\mathrm{C} 23 & -157.64(12) \\ \mathrm{C} 41-\mathrm{C} 25 \mathrm{~A}-\mathrm{C} 30 \mathrm{~A}-\mathrm{C} 23 & 76.86(16) \\ \mathrm{C} 25-\mathrm{C} 25 \mathrm{~A}-\mathrm{C} 30 \mathrm{~A}-\mathrm{C} 23 & -40.30(14) \\ \mathrm{C} 25 \mathrm{~B}-\mathrm{C} 25 \mathrm{~A}-\mathrm{C} 30 \mathrm{~A}-\mathrm{C} 30 & -38.65(15) \\ \mathrm{C} 41-\mathrm{C} 25 \mathrm{~A}-\mathrm{C} 30 \mathrm{~A}-\mathrm{C} 30 & -164.14(13) \\ \mathrm{C} 25-\mathrm{C} 25 \mathrm{~A}-\mathrm{C} 30 \mathrm{~A}-\mathrm{C} 30 & 78.70(13) \\ \mathrm{C} 29 \mathrm{~A}-\mathrm{C} 30-\mathrm{C} 30 \mathrm{~A}-\mathrm{C} 23 & 151.59(13) \\ \mathrm{C} 31-\mathrm{C} 30-\mathrm{C} 30 \mathrm{~A}-\mathrm{C} 23 & 37.56(17) \\ \mathrm{C} 29 \mathrm{~A}-\mathrm{C} 30-\mathrm{C} 30 \mathrm{~A}-\mathrm{C} 25 \mathrm{~A} & 40.86(14) \\ \mathrm{C} 31-\mathrm{C} 30-\mathrm{C} 30 \mathrm{~A}-\mathrm{C} 25 \mathrm{~A} & -73.17(14) \\ \mathrm{C} 29 \mathrm{~A}-\mathrm{C} 30-\mathrm{C} 31-\mathrm{N} 32 & -54.65(16) \\ \mathrm{C} 30 \mathrm{~A}-\mathrm{C} 30-\mathrm{C} 31-\mathrm{N} 32 & 54.58(16) \\ \mathrm{C} 30-\mathrm{C} 31-\mathrm{N} 32-\mathrm{C} 44 & -172.92(12) \\ \mathrm{C} 30-\mathrm{C} 31-\mathrm{N} 32-\mathrm{C} 25 & -46.02(16) \\ \mathrm{C} 24-\mathrm{C} 25-\mathrm{N} 32-\mathrm{C} 44 & 73.76(16) \\ \mathrm{C} 25 \mathrm{~A}-\mathrm{C} 25-\mathrm{N} 32-\mathrm{C} 44 & -175.20(12) \\ \mathrm{C} 24-\mathrm{C} 25-\mathrm{N} 32-\mathrm{C} 31 & -52.44(16) \\ \mathrm{C} 25 \mathrm{~A}-\mathrm{C} 25-\mathrm{N} 32-\mathrm{C} 31 & 58.60(16) \\ \mathrm{C} 24-\mathrm{C} 23-\mathrm{C} 33-\mathrm{C} 34 & -43.9(3)\end{array}$




\begin{tabular}{|c|c|c|c|}
\hline $\mathrm{C} 3 \mathrm{~B}-\mathrm{C} 4-\mathrm{C} 5-\mathrm{C} 6$ & $2.6(2)$ & $\mathrm{C} 30 \mathrm{~A}-\mathrm{C} 23-\mathrm{C} 33-\mathrm{C} 34$ & $139.35(17)$ \\
\hline $\mathrm{C} 20-\mathrm{O} 4-\mathrm{C} 6-\mathrm{C} 7$ & $12.3(2)$ & $\mathrm{C} 24-\mathrm{C} 23-\mathrm{C} 33-\mathrm{C} 38$ & $137.56(19)$ \\
\hline $\mathrm{C} 20-\mathrm{O} 4-\mathrm{C} 6-\mathrm{C} 5$ & $-168.59(14)$ & $\mathrm{C} 30 \mathrm{~A}-\mathrm{C} 23-\mathrm{C} 33-\mathrm{C} 38$ & $-39.2(2)$ \\
\hline $\mathrm{O} 3-\mathrm{C} 5-\mathrm{C} 6-\mathrm{O} 4$ & $-2.0(2)$ & $\mathrm{C} 38-\mathrm{C} 33-\mathrm{C} 34-\mathrm{C} 35$ & $-1.7(3)$ \\
\hline $\mathrm{C} 4-\mathrm{C} 5-\mathrm{C} 6-\mathrm{O} 4$ & $177.74(14)$ & $\mathrm{C} 23-\mathrm{C} 33-\mathrm{C} 34-\mathrm{C} 35$ & $179.80(17)$ \\
\hline $\mathrm{O} 3-\mathrm{C} 5-\mathrm{C} 6-\mathrm{C} 7$ & $177.21(14)$ & $\mathrm{C} 33-\mathrm{C} 34-\mathrm{C} 35-\mathrm{C} 36$ & $-0.5(3)$ \\
\hline $\mathrm{C} 4-\mathrm{C} 5-\mathrm{C} 6-\mathrm{C} 7$ & $-3.1(2)$ & C34-C35-C36-C37 & $1.1(3)$ \\
\hline $\mathrm{O} 4-\mathrm{C} 6-\mathrm{C} 7-\mathrm{C} 7 \mathrm{~A}$ & $179.67(14)$ & $\mathrm{C} 35-\mathrm{C} 36-\mathrm{C} 37-\mathrm{C} 38$ & $0.4(3)$ \\
\hline $\mathrm{C} 5-\mathrm{C} 6-\mathrm{C} 7-\mathrm{C} 7 \mathrm{~A}$ & $0.6(2)$ & $\mathrm{C} 36-\mathrm{C} 37-\mathrm{C} 38-\mathrm{C} 33$ & $-2.7(3)$ \\
\hline $\mathrm{C} 4-\mathrm{C} 3 \mathrm{~B}-\mathrm{C} 7 \mathrm{~A}-\mathrm{C} 7$ & $-2.9(2)$ & $\mathrm{C} 34-\mathrm{C} 33-\mathrm{C} 38-\mathrm{C} 37$ & $3.2(3)$ \\
\hline $\mathrm{C} 3 \mathrm{~A}-\mathrm{C} 3 \mathrm{~B}-\mathrm{C} 7 \mathrm{~A}-\mathrm{C} 7$ & $177.10(14)$ & $\mathrm{C} 23-\mathrm{C} 33-\mathrm{C} 38-\mathrm{C} 37$ & $-178.16(16)$ \\
\hline $\mathrm{C} 4-\mathrm{C} 3 \mathrm{~B}-\mathrm{C} 7 \mathrm{~A}-\mathrm{C} 8$ & $-177.06(14)$ & $\mathrm{C} 40-\mathrm{O} 6-\mathrm{C} 39-\mathrm{O} 5$ & $-3.2(2)$ \\
\hline $\mathrm{C} 3 \mathrm{~A}-\mathrm{C} 3 \mathrm{~B}-\mathrm{C} 7 \mathrm{~A}-\mathrm{C} 8$ & $2.94(18)$ & $\mathrm{C} 40-\mathrm{O} 6-\mathrm{C} 39-\mathrm{C} 24$ & $176.56(15)$ \\
\hline $\mathrm{C} 6-\mathrm{C} 7-\mathrm{C} 7 \mathrm{~A}-\mathrm{C} 3 \mathrm{~B}$ & $2.4(2)$ & $\mathrm{C} 23-\mathrm{C} 24-\mathrm{C} 39-\mathrm{O} 5$ & $165.36(17)$ \\
\hline $\mathrm{C} 6-\mathrm{C} 7-\mathrm{C} 7 \mathrm{~A}-\mathrm{C} 8$ & $175.21(15)$ & $\mathrm{C} 25-\mathrm{C} 24-\mathrm{C} 39-\mathrm{O} 5$ & $-22.9(2)$ \\
\hline $\mathrm{C} 3 \mathrm{~B}-\mathrm{C} 7 \mathrm{~A}-\mathrm{C} 8-\mathrm{C} 9$ & $86.25(16)$ & $\mathrm{C} 23-\mathrm{C} 24-\mathrm{C} 39-\mathrm{O} 6$ & $-14.4(3)$ \\
\hline $\mathrm{C} 7-\mathrm{C} 7 \mathrm{~A}-\mathrm{C} 8-\mathrm{C} 9$ & $-87.25(19)$ & $\mathrm{C} 25-\mathrm{C} 24-\mathrm{C} 39-\mathrm{O} 6$ & $157.40(14)$ \\
\hline $\mathrm{C} 3 \mathrm{~B}-\mathrm{C} 7 \mathrm{~A}-\mathrm{C} 8-\mathrm{C} 8 \mathrm{~A}$ & $-27.78(16)$ & $\mathrm{C} 55-\mathrm{C} 45-\mathrm{C} 46-\mathrm{C} 61$ & $-2.5(3)$ \\
\hline $\mathrm{C} 7-\mathrm{C} 7 \mathrm{~A}-\mathrm{C} 8-\mathrm{C} 8 \mathrm{~A}$ & $158.72(16)$ & $\mathrm{C} 52 \mathrm{~A}-\mathrm{C} 45-\mathrm{C} 46-\mathrm{C} 61$ & $177.20(16)$ \\
\hline $\mathrm{C} 2-\mathrm{C} 1-\mathrm{C} 8 \mathrm{~A}-\mathrm{C} 3 \mathrm{~A}$ & $25.11(17)$ & $\mathrm{C} 55-\mathrm{C} 45-\mathrm{C} 46-\mathrm{C} 47$ & $-175.48(16)$ \\
\hline $\mathrm{C} 11-\mathrm{C} 1-\mathrm{C} 8 \mathrm{~A}-\mathrm{C} 3 \mathrm{~A}$ & $-153.63(14)$ & $\mathrm{C} 52 \mathrm{~A}-\mathrm{C} 45-\mathrm{C} 46-\mathrm{C} 47$ & $4.22(18)$ \\
\hline $\mathrm{C} 2-\mathrm{C} 1-\mathrm{C} 8 \mathrm{~A}-\mathrm{C} 8$ & $-83.04(17)$ & $\mathrm{C} 45-\mathrm{C} 46-\mathrm{C} 47-\mathrm{N} 54$ & $85.56(16)$ \\
\hline $\mathrm{C} 11-\mathrm{C} 1-\mathrm{C} 8 \mathrm{~A}-\mathrm{C} 8$ & $98.23(17)$ & $\mathrm{C} 61-\mathrm{C} 46-\mathrm{C} 47-\mathrm{N} 54$ & $-88.48(17)$ \\
\hline $\mathrm{C} 3 \mathrm{~B}-\mathrm{C} 3 \mathrm{~A}-\mathrm{C} 8 \mathrm{~A}-\mathrm{C} 1$ & $-158.40(13)$ & $\mathrm{C} 45-\mathrm{C} 46-\mathrm{C} 47-\mathrm{C} 47 \mathrm{~A}$ & $-31.37(17)$ \\
\hline $\mathrm{C} 19-\mathrm{C} 3 \mathrm{~A}-\mathrm{C} 8 \mathrm{~A}-\mathrm{C} 1$ & $76.66(16)$ & $\mathrm{C} 61-\mathrm{C} 46-\mathrm{C} 47-\mathrm{C} 47 \mathrm{~A}$ & $154.60(14)$ \\
\hline $\mathrm{C} 3-\mathrm{C} 3 \mathrm{~A}-\mathrm{C} 8 \mathrm{~A}-\mathrm{C} 1$ & $-41.65(14)$ & N54-C47-C47A-C47B & $32.61(18)$ \\
\hline $\mathrm{C} 3 \mathrm{~B}-\mathrm{C} 3 \mathrm{~A}-\mathrm{C} 8 \mathrm{~A}-\mathrm{C} 8$ & $-38.74(14)$ & $\mathrm{C} 46-\mathrm{C} 47-\mathrm{C} 47 \mathrm{~A}-\mathrm{C} 47 \mathrm{~B}$ & $149.97(13)$ \\
\hline $\mathrm{C} 19-\mathrm{C} 3 \mathrm{~A}-\mathrm{C} 8 \mathrm{~A}-\mathrm{C} 8$ & $-163.67(13)$ & N54-C47-C47A-C63 & $164.35(13)$ \\
\hline $\mathrm{C} 3-\mathrm{C} 3 \mathrm{~A}-\mathrm{C} 8 \mathrm{~A}-\mathrm{C} 8$ & $78.01(13)$ & $\mathrm{C} 46-\mathrm{C} 47-\mathrm{C} 47 \mathrm{~A}-\mathrm{C} 63$ & $-78.29(15)$ \\
\hline $\mathrm{C} 7 \mathrm{~A}-\mathrm{C} 8-\mathrm{C} 8 \mathrm{~A}-\mathrm{C} 1$ & $149.93(13)$ & N54-C47-C47A-C52A & $-74.25(14)$ \\
\hline $\mathrm{C} 9-\mathrm{C} 8-\mathrm{C} 8 \mathrm{~A}-\mathrm{C} 1$ & $36.53(18)$ & $\mathrm{C} 46-\mathrm{C} 47-\mathrm{C} 47 \mathrm{~A}-\mathrm{C} 52 \mathrm{~A}$ & $43.12(14)$ \\
\hline $\mathrm{C} 7 \mathrm{~A}-\mathrm{C} 8-\mathrm{C} 8 \mathrm{~A}-\mathrm{C} 3 \mathrm{~A}$ & $40.12(14)$ & $\mathrm{C} 63-\mathrm{C} 47 \mathrm{~A}-\mathrm{C} 47 \mathrm{~B}-\mathrm{C} 51 \mathrm{~A}$ & $150.67(14)$ \\
\hline $\mathrm{C} 9-\mathrm{C} 8-\mathrm{C} 8 \mathrm{~A}-\mathrm{C} 3 \mathrm{~A}$ & $-73.29(15)$ & $\mathrm{C} 52 \mathrm{~A}-\mathrm{C} 47 \mathrm{~A}-\mathrm{C} 47 \mathrm{~B}-\mathrm{C} 51 \mathrm{~A}$ & $23.74(17)$ \\
\hline $\mathrm{C} 7 \mathrm{~A}-\mathrm{C} 8-\mathrm{C} 9-\mathrm{N} 10$ & $-53.83(16)$ & $\mathrm{C} 47-\mathrm{C} 47 \mathrm{~A}-\mathrm{C} 47 \mathrm{~B}-\mathrm{C} 51 \mathrm{~A}$ & $-79.52(17)$ \\
\hline $\mathrm{C} 8 \mathrm{~A}-\mathrm{C} 8-\mathrm{C} 9-\mathrm{N} 10$ & $55.13(16)$ & $\mathrm{C} 63-\mathrm{C} 47 \mathrm{~A}-\mathrm{C} 47 \mathrm{~B}-\mathrm{C} 48$ & $-31.1(2)$ \\
\hline $\mathrm{C} 8-\mathrm{C} 9-\mathrm{N} 10-\mathrm{C} 22$ & $-174.44(12)$ & $\mathrm{C} 52 \mathrm{~A}-\mathrm{C} 47 \mathrm{~A}-\mathrm{C} 47 \mathrm{~B}-\mathrm{C} 48$ & $-158.03(17)$ \\
\hline $\mathrm{C} 8-\mathrm{C} 9-\mathrm{N} 10-\mathrm{C} 3$ & $-46.77(16)$ & $\mathrm{C} 47-\mathrm{C} 47 \mathrm{~A}-\mathrm{C} 47 \mathrm{~B}-\mathrm{C} 48$ & $98.7(2)$ \\
\hline $\mathrm{C} 2-\mathrm{C} 3-\mathrm{N} 10-\mathrm{C} 22$ & $74.47(16)$ & $\mathrm{C} 51 \mathrm{~A}-\mathrm{C} 47 \mathrm{~B}-\mathrm{C} 48-\mathrm{C} 49$ & $1.2(2)$ \\
\hline $\mathrm{C} 3 \mathrm{~A}-\mathrm{C} 3-\mathrm{N} 10-\mathrm{C} 22$ & $-174.60(12)$ & $\mathrm{C} 47 \mathrm{~A}-\mathrm{C} 47 \mathrm{~B}-\mathrm{C} 48-\mathrm{C} 49$ & $-176.84(16)$ \\
\hline $\mathrm{C} 2-\mathrm{C} 3-\mathrm{N} 10-\mathrm{C} 9$ & $-51.88(17)$ & $\mathrm{C} 65-\mathrm{O} 11-\mathrm{C} 49-\mathrm{C} 48$ & $9.1(2)$ \\
\hline $\mathrm{C} 3 \mathrm{~A}-\mathrm{C} 3-\mathrm{N} 10-\mathrm{C} 9$ & $59.06(16)$ & $\mathrm{C} 65-\mathrm{O} 11-\mathrm{C} 49-\mathrm{C} 50$ & $-171.75(15)$ \\
\hline $\mathrm{C} 2-\mathrm{C} 1-\mathrm{C} 11-\mathrm{C} 12$ & $-48.2(3)$ & $\mathrm{C} 47 \mathrm{~B}-\mathrm{C} 48-\mathrm{C} 49-\mathrm{O} 11$ & $-178.54(15)$ \\
\hline $\mathrm{C} 8 \mathrm{~A}-\mathrm{C} 1-\mathrm{C} 11-\mathrm{C} 12$ & $130.16(17)$ & $\mathrm{C} 47 \mathrm{~B}-\mathrm{C} 48-\mathrm{C} 49-\mathrm{C} 50$ & $2.4(2)$ \\
\hline $\mathrm{C} 2-\mathrm{C} 1-\mathrm{C} 11-\mathrm{C} 16$ & $134.47(19)$ & $\mathrm{C} 64-\mathrm{O} 12-\mathrm{C} 50-\mathrm{C} 51$ & $13.6(3)$ \\
\hline $\mathrm{C} 8 \mathrm{~A}-\mathrm{C} 1-\mathrm{C} 11-\mathrm{C} 16$ & $-47.1(2)$ & $\mathrm{C} 64-\mathrm{O} 12-\mathrm{C} 50-\mathrm{C} 49$ & $-165.73(16)$ \\
\hline $\mathrm{C} 16-\mathrm{C} 11-\mathrm{C} 12-\mathrm{C} 13$ & $-1.0(3)$ & $\mathrm{O} 11-\mathrm{C} 49-\mathrm{C} 50-\mathrm{O} 12$ & $-3.4(2)$ \\
\hline $\mathrm{C} 1-\mathrm{C} 11-\mathrm{C} 12-\mathrm{C} 13$ & $-178.31(17)$ & $\mathrm{C} 48-\mathrm{C} 49-\mathrm{C} 50-\mathrm{O} 12$ & $175.73(15)$ \\
\hline $\mathrm{C} 11-\mathrm{C} 12-\mathrm{C} 13-\mathrm{C} 14$ & $-0.9(3)$ & $\mathrm{O} 11-\mathrm{C} 49-\mathrm{C} 50-\mathrm{C} 51$ & $177.20(15)$ \\
\hline
\end{tabular}




\begin{tabular}{|c|c|c|c|}
\hline $\mathrm{C} 12-\mathrm{C} 13-\mathrm{C} 14-\mathrm{C} 15$ & $1.3(3)$ & $\mathrm{C} 48-\mathrm{C} 49-\mathrm{C} 50-\mathrm{C} 51$ & $-3.6(3)$ \\
\hline $\mathrm{C} 13-\mathrm{C} 14-\mathrm{C} 15-\mathrm{C} 16$ & $0.2(3)$ & $\mathrm{O} 12-\mathrm{C} 50-\mathrm{C} 51-\mathrm{C} 51 \mathrm{~A}$ & $-178.05(15)$ \\
\hline $\mathrm{C} 14-\mathrm{C} 15-\mathrm{C} 16-\mathrm{C} 11$ & $-2.2(3)$ & $\mathrm{C} 49-\mathrm{C} 50-\mathrm{C} 51-\mathrm{C} 51 \mathrm{~A}$ & $1.2(3)$ \\
\hline $\mathrm{C} 12-\mathrm{C} 11-\mathrm{C} 16-\mathrm{C} 15$ & $2.5(3)$ & $\mathrm{C} 48-\mathrm{C} 47 \mathrm{~B}-\mathrm{C} 51 \mathrm{~A}-\mathrm{C} 51$ & $-3.6(3)$ \\
\hline $\mathrm{C} 1-\mathrm{C} 11-\mathrm{C} 16-\mathrm{C} 15$ & $179.93(16)$ & $\mathrm{C} 47 \mathrm{~A}-\mathrm{C} 47 \mathrm{~B}-\mathrm{C} 51 \mathrm{~A}-\mathrm{C} 51$ & $174.76(15)$ \\
\hline $\mathrm{C} 18-\mathrm{O} 2-\mathrm{C} 17-\mathrm{O} 1$ & $-2.2(2)$ & $\mathrm{C} 48-\mathrm{C} 47 \mathrm{~B}-\mathrm{C} 51 \mathrm{~A}-\mathrm{C} 52$ & $-176.13(15)$ \\
\hline $\mathrm{C} 18-\mathrm{O} 2-\mathrm{C} 17-\mathrm{C} 2$ & $177.75(15)$ & $\mathrm{C} 47 \mathrm{~A}-\mathrm{C} 47 \mathrm{~B}-\mathrm{C} 51 \mathrm{~A}-\mathrm{C} 52$ & $2.28(18)$ \\
\hline $\mathrm{C} 1-\mathrm{C} 2-\mathrm{C} 17-\mathrm{O} 1$ & $172.56(18)$ & $\mathrm{C} 50-\mathrm{C} 51-\mathrm{C} 51 \mathrm{~A}-\mathrm{C} 47 \mathrm{~B}$ & $2.4(2)$ \\
\hline $\mathrm{C} 3-\mathrm{C} 2-\mathrm{C} 17-\mathrm{O} 1$ & $-14.8(2)$ & $\mathrm{C} 50-\mathrm{C} 51-\mathrm{C} 51 \mathrm{~A}-\mathrm{C} 52$ & $173.18(16)$ \\
\hline $\mathrm{C} 1-\mathrm{C} 2-\mathrm{C} 17-\mathrm{O} 2$ & $-7.4(3)$ & $\mathrm{C} 47 \mathrm{~B}-\mathrm{C} 51 \mathrm{~A}-\mathrm{C} 52-\mathrm{C} 53$ & $86.89(16)$ \\
\hline $\mathrm{C} 3-\mathrm{C} 2-\mathrm{C} 17-\mathrm{O} 2$ & $165.25(14)$ & $\mathrm{C} 51-\mathrm{C} 51 \mathrm{~A}-\mathrm{C} 52-\mathrm{C} 53$ & $-84.8(2)$ \\
\hline $\mathrm{C} 33-\mathrm{C} 23-\mathrm{C} 24-\mathrm{C} 39$ & $0.6(3)$ & $\mathrm{C} 47 \mathrm{~B}-\mathrm{C} 51 \mathrm{~A}-\mathrm{C} 52-\mathrm{C} 52 \mathrm{~A}$ & $-27.06(17)$ \\
\hline $\mathrm{C} 30 \mathrm{~A}-\mathrm{C} 23-\mathrm{C} 24-\mathrm{C} 39$ & $177.66(16)$ & $\mathrm{C} 51-\mathrm{C} 51 \mathrm{~A}-\mathrm{C} 52-\mathrm{C} 52 \mathrm{~A}$ & $161.30(17)$ \\
\hline $\mathrm{C} 33-\mathrm{C} 23-\mathrm{C} 24-\mathrm{C} 25$ & $-171.75(16)$ & $\mathrm{C} 46-\mathrm{C} 45-\mathrm{C} 52 \mathrm{~A}-\mathrm{C} 47 \mathrm{~A}$ & $24.97(17)$ \\
\hline $\mathrm{C} 30 \mathrm{~A}-\mathrm{C} 23-\mathrm{C} 24-\mathrm{C} 25$ & $5.29(18)$ & $\mathrm{C} 55-\mathrm{C} 45-\mathrm{C} 52 \mathrm{~A}-\mathrm{C} 47 \mathrm{~A}$ & $-155.29(14)$ \\
\hline $\mathrm{C} 23-\mathrm{C} 24-\mathrm{C} 25-\mathrm{N} 32$ & $84.75(16)$ & $\mathrm{C} 46-\mathrm{C} 45-\mathrm{C} 52 \mathrm{~A}-\mathrm{C} 52$ & $-83.22(17)$ \\
\hline $\mathrm{C} 39-\mathrm{C} 24-\mathrm{C} 25-\mathrm{N} 32$ & $-88.66(17)$ & $\mathrm{C} 55-\mathrm{C} 45-\mathrm{C} 52 \mathrm{~A}-\mathrm{C} 52$ & $96.52(17)$ \\
\hline $\mathrm{C} 23-\mathrm{C} 24-\mathrm{C} 25-\mathrm{C} 25 \mathrm{~A}$ & $-31.61(16)$ & $\mathrm{C} 47 \mathrm{~B}-\mathrm{C} 47 \mathrm{~A}-\mathrm{C} 52 \mathrm{~A}-\mathrm{C} 45$ & $-157.99(13)$ \\
\hline $\mathrm{C} 39-\mathrm{C} 24-\mathrm{C} 25-\mathrm{C} 25 \mathrm{~A}$ & $154.98(14)$ & $\mathrm{C} 63-\mathrm{C} 47 \mathrm{~A}-\mathrm{C} 52 \mathrm{~A}-\mathrm{C} 45$ & $75.88(17)$ \\
\hline $\mathrm{N} 32-\mathrm{C} 25-\mathrm{C} 25 \mathrm{~A}-\mathrm{C} 25 \mathrm{~B}$ & $32.49(18)$ & $\mathrm{C} 47-\mathrm{C} 47 \mathrm{~A}-\mathrm{C} 52 \mathrm{~A}-\mathrm{C} 45$ & $-41.51(14)$ \\
\hline $\mathrm{C} 24-\mathrm{C} 25-\mathrm{C} 25 \mathrm{~A}-\mathrm{C} 25 \mathrm{~B}$ & $149.30(13)$ & $\mathrm{C} 47 \mathrm{~B}-\mathrm{C} 47 \mathrm{~A}-\mathrm{C} 52 \mathrm{~A}-\mathrm{C} 52$ & $-38.55(15)$ \\
\hline $\mathrm{N} 32-\mathrm{C} 25-\mathrm{C} 25 \mathrm{~A}-\mathrm{C} 41$ & $163.65(12)$ & $\mathrm{C} 63-\mathrm{C} 47 \mathrm{~A}-\mathrm{C} 52 \mathrm{~A}-\mathrm{C} 52$ & $-164.68(13)$ \\
\hline $\mathrm{C} 24-\mathrm{C} 25-\mathrm{C} 25 \mathrm{~A}-\mathrm{C} 41$ & $-79.54(15)$ & $\mathrm{C} 47-\mathrm{C} 47 \mathrm{~A}-\mathrm{C} 52 \mathrm{~A}-\mathrm{C} 52$ & $77.94(13)$ \\
\hline $\mathrm{N} 32-\mathrm{C} 25-\mathrm{C} 25 \mathrm{~A}-\mathrm{C} 30 \mathrm{~A}$ & $-74.56(14)$ & $\mathrm{C} 51 \mathrm{~A}-\mathrm{C} 52-\mathrm{C} 52 \mathrm{~A}-\mathrm{C} 45$ & $149.79(13)$ \\
\hline $\mathrm{C} 24-\mathrm{C} 25-\mathrm{C} 25 \mathrm{~A}-\mathrm{C} 30 \mathrm{~A}$ & $42.26(13)$ & $\mathrm{C} 53-\mathrm{C} 52-\mathrm{C} 52 \mathrm{~A}-\mathrm{C} 45$ & $36.38(18)$ \\
\hline $\mathrm{C} 41-\mathrm{C} 25 \mathrm{~A}-\mathrm{C} 25 \mathrm{~B}-\mathrm{C} 26$ & $-29.3(2)$ & $\mathrm{C} 51 \mathrm{~A}-\mathrm{C} 52-\mathrm{C} 52 \mathrm{~A}-\mathrm{C} 47 \mathrm{~A}$ & $39.48(15)$ \\
\hline $\mathrm{C} 30 \mathrm{~A}-\mathrm{C} 25 \mathrm{~A}-\mathrm{C} 25 \mathrm{~B}-\mathrm{C} 26$ & $-156.20(16)$ & $\mathrm{C} 53-\mathrm{C} 52-\mathrm{C} 52 \mathrm{~A}-\mathrm{C} 47 \mathrm{~A}$ & $-73.93(15)$ \\
\hline $\mathrm{C} 25-\mathrm{C} 25 \mathrm{~A}-\mathrm{C} 25 \mathrm{~B}-\mathrm{C} 26$ & $100.0(2)$ & $\mathrm{C} 51 \mathrm{~A}-\mathrm{C} 52-\mathrm{C} 53-\mathrm{N} 54$ & $-53.32(16)$ \\
\hline $\mathrm{C} 41-\mathrm{C} 25 \mathrm{~A}-\mathrm{C} 25 \mathrm{~B}-\mathrm{C} 29 \mathrm{~A}$ & $149.53(14)$ & $\mathrm{C} 52 \mathrm{~A}-\mathrm{C} 52-\mathrm{C} 53-\mathrm{N} 54$ & $55.67(16)$ \\
\hline $\mathrm{C} 30 \mathrm{~A}-\mathrm{C} 25 \mathrm{~A}-\mathrm{C} 25 \mathrm{~B}-\mathrm{C} 29 \mathrm{~A}$ & $22.64(17)$ & $\mathrm{C} 52-\mathrm{C} 53-\mathrm{N} 54-\mathrm{C} 66$ & $-174.61(12)$ \\
\hline $\mathrm{C} 25-\mathrm{C} 25 \mathrm{~A}-\mathrm{C} 25 \mathrm{~B}-\mathrm{C} 29 \mathrm{~A}$ & $-81.20(17)$ & $\mathrm{C} 52-\mathrm{C} 53-\mathrm{N} 54-\mathrm{C} 47$ & $-47.09(17)$ \\
\hline $\mathrm{C} 29 \mathrm{~A}-\mathrm{C} 25 \mathrm{~B}-\mathrm{C} 26-\mathrm{C} 27$ & $-1.6(2)$ & $\mathrm{C} 46-\mathrm{C} 47-\mathrm{N} 54-\mathrm{C} 66$ & $74.48(16)$ \\
\hline $\mathrm{C} 25 \mathrm{~A}-\mathrm{C} 25 \mathrm{~B}-\mathrm{C} 26-\mathrm{C} 27$ & $177.10(15)$ & $\mathrm{C} 47 \mathrm{~A}-\mathrm{C} 47-\mathrm{N} 54-\mathrm{C} 66$ & $-174.19(13)$ \\
\hline $\mathrm{C} 43-\mathrm{O} 7-\mathrm{C} 27-\mathrm{C} 26$ & $14.1(2)$ & $\mathrm{C} 46-\mathrm{C} 47-\mathrm{N} 54-\mathrm{C} 53$ & $-52.04(17)$ \\
\hline $\mathrm{C} 43-\mathrm{O} 7-\mathrm{C} 27-\mathrm{C} 28$ & $-165.90(15)$ & $\mathrm{C} 47 \mathrm{~A}-\mathrm{C} 47-\mathrm{N} 54-\mathrm{C} 53$ & $59.29(16)$ \\
\hline $\mathrm{C} 25 \mathrm{~B}-\mathrm{C} 26-\mathrm{C} 27-\mathrm{O} 7$ & $-178.15(15)$ & $\mathrm{C} 46-\mathrm{C} 45-\mathrm{C} 55-\mathrm{C} 56$ & $-42.3(3)$ \\
\hline $\mathrm{C} 25 \mathrm{~B}-\mathrm{C} 26-\mathrm{C} 27-\mathrm{C} 28$ & $1.9(2)$ & $\mathrm{C} 52 \mathrm{~A}-\mathrm{C} 45-\mathrm{C} 55-\mathrm{C} 56$ & $138.03(17)$ \\
\hline $\mathrm{C} 42-\mathrm{O} 8-\mathrm{C} 28-\mathrm{C} 29$ & $103.54(18)$ & $\mathrm{C} 46-\mathrm{C} 45-\mathrm{C} 55-\mathrm{C} 60$ & $140.13(19)$ \\
\hline $\mathrm{C} 42-\mathrm{O} 8-\mathrm{C} 28-\mathrm{C} 27$ & $-81.8(2)$ & $\mathrm{C} 52 \mathrm{~A}-\mathrm{C} 45-\mathrm{C} 55-\mathrm{C} 60$ & $-39.5(2)$ \\
\hline $\mathrm{O} 7-\mathrm{C} 27-\mathrm{C} 28-\mathrm{C} 29$ & $179.56(15)$ & $\mathrm{C} 60-\mathrm{C} 55-\mathrm{C} 56-\mathrm{C} 57$ & $-1.1(3)$ \\
\hline $\mathrm{C} 26-\mathrm{C} 27-\mathrm{C} 28-\mathrm{C} 29$ & $-0.5(2)$ & $\mathrm{C} 45-\mathrm{C} 55-\mathrm{C} 56-\mathrm{C} 57$ & $-178.70(16)$ \\
\hline $\mathrm{O} 7-\mathrm{C} 27-\mathrm{C} 28-\mathrm{O} 8$ & $5.0(2)$ & $\mathrm{C} 55-\mathrm{C} 56-\mathrm{C} 57-\mathrm{C} 58$ & $-0.7(3)$ \\
\hline $\mathrm{C} 26-\mathrm{C} 27-\mathrm{C} 28-\mathrm{O} 8$ & $-175.00(14)$ & $\mathrm{C} 56-\mathrm{C} 57-\mathrm{C} 58-\mathrm{C} 59$ & $1.0(3)$ \\
\hline $\mathrm{O} 8-\mathrm{C} 28-\mathrm{C} 29-\mathrm{C} 29 \mathrm{~A}$ & $173.37(14)$ & $\mathrm{C} 57-\mathrm{C} 58-\mathrm{C} 59-\mathrm{C} 60$ & $0.5(3)$ \\
\hline $\mathrm{C} 27-\mathrm{C} 28-\mathrm{C} 29-\mathrm{C} 29 \mathrm{~A}$ & $-1.2(2)$ & $\mathrm{C} 58-\mathrm{C} 59-\mathrm{C} 60-\mathrm{C} 55$ & $-2.4(3)$ \\
\hline $\mathrm{C} 28-\mathrm{C} 29-\mathrm{C} 29 \mathrm{~A}-\mathrm{C} 25 \mathrm{~B}$ & $1.5(2)$ & $\mathrm{C} 56-\mathrm{C} 55-\mathrm{C} 60-\mathrm{C} 59$ & $2.7(2)$ \\
\hline $\mathrm{C} 28-\mathrm{C} 29-\mathrm{C} 29 \mathrm{~A}-\mathrm{C} 30$ & $177.52(16)$ & $\mathrm{C} 45-\mathrm{C} 55-\mathrm{C} 60-\mathrm{C} 59$ & $-179.63(15)$ \\
\hline $\mathrm{C} 26-\mathrm{C} 25 \mathrm{~B}-\mathrm{C} 29 \mathrm{~A}-\mathrm{C} 29$ & $-0.1(2)$ & $\mathrm{C} 62-\mathrm{O} 10-\mathrm{C} 61-\mathrm{O} 9$ & $-4.4(2)$ \\
\hline
\end{tabular}




$\begin{array}{llll}\mathrm{C} 25 \mathrm{~A}-\mathrm{C} 25 \mathrm{~B}-\mathrm{C} 29 \mathrm{~A}-\mathrm{C} 29 & -179.06(15) & \mathrm{C} 62-\mathrm{O} 10-\mathrm{C} 61-\mathrm{C} 46 & 175.83(14) \\ \mathrm{C} 26-\mathrm{C} 25 \mathrm{~B}-\mathrm{C} 29 \mathrm{~A}-\mathrm{C} 30 & -176.84(14) & \mathrm{C} 45-\mathrm{C} 46-\mathrm{C} 61-\mathrm{O} 9 & 168.95(17) \\ \mathrm{C} 25 \mathrm{~A}-\mathrm{C} 25 \mathrm{~B}-\mathrm{C} 29 \mathrm{~A}-\mathrm{C} 30 & 4.20(18) & \mathrm{C} 47-\mathrm{C} 46-\mathrm{C} 61-\mathrm{O} 9 & -18.6(2) \\ \mathrm{C} 29-\mathrm{C} 29 \mathrm{~A}-\mathrm{C} 30-\mathrm{C} 31 & -91.4(2) & \mathrm{C} 45-\mathrm{C} 46-\mathrm{C} 61-\mathrm{O} 10 & -11.3(3) \\ \mathrm{C} 25 \mathrm{~B}-\mathrm{C} 29 \mathrm{~A}-\mathrm{C} 30-\mathrm{C} 31 & 84.96(16) & \mathrm{C} 47-\mathrm{C} 46-\mathrm{C} 61-\mathrm{O} 10 & 161.13(14)\end{array}$

